\title{
Ellipsoidal Sets for Resilient and Robust Static Output-feedback
}

\author{
Dimitri Peaucelle - Denis Arzelier \\ $L A A S$ - CNRS \\ 7, Avenue du Colonel Roche - 31077 Toulouse CEDEX 4 - FRANCE \\ Tel. 0561336309 fax: 0561336969 \\ email:peaucelle@laas.fr - arzelier@laas.fr
}

\begin{abstract}
The robust static output-feedback synthesis for linear time invariant systems is considered. The static output-feedback design is shown to have analogies with robust analysis, in particular with the existence of some quadratic separator. These considerations lead to formulate the problem as the synthesis of an ellipsoidal set of stabilising controllers. The designed stabilising set is shown to guarantee the resilience with respect to uncertain control law implementation. Several results are then derived for robust stabilisability with respect to rational-dissipative uncertainty models. Additionally, extensions to robust performances are given for robust pole location, robust $H_{\infty}$ and robust $H_{2}$ objectives. As a conclusion, the paper brings out a global approach to robust static output-feedback design where multiple specifications can be simultaneously defined without increasing the global complexity.
\end{abstract}

\section{Keywords}

Output-feedback, Quadratic Separation, Fragility, Robustness, pole location, $H_{2}, H_{\infty}$, Multi-Objective, LMI.

\section{INTRODUCTION}

One of the most challenging open problems in control theory is the synthesis of fixed-order or static outputfeedback controllers that meet desired performances and robustness specifications [BER 92], [BLO 95], [SYR 97]. Among all possible varieties of this open problem, the introduction first explicits the adopted theoretical framework; then, the novel ellipsoidal design is described; at last, this design is shown to overcome the existing techniques.

\section{A. Framework}

The Static Output-feedback (SOF) synthesis problem implies, for a given class of systems, to derive theoretical conditions for the existence of a static control law and associate these with numerical methods. The adopted modelling, theoretical and computational frameworks are first exposed. Then, the complexity of the adopted SOF problem is formulated.

Modelling: The class of continuous-time, Linear Time-Invariant (LTI) systems is addressed. The systems are given as multi-input/multi-output state-space models. In addition to the control input vector and the measure output vector that define the control loop, the models may include some other input/output signals. These are introduced for input/output performance specifications defined by $H_{2}$ and $H_{\infty}$ norms. In addition, the LTI systems are assumed to be affected by rational dissipative parametric uncertainty [PEA 98a], [XIE 98].

Lyapunov theory and LMIs: The theoretical framework is based on the Lyapunov theory and the results are formulated with the help of Linear Matrix Inequalities (LMIs). A great number of problems regarding automatic control theory with respect to linear systems have been formulated within this scheme (see the books [BOY 94], [ELG 00] for surveys). Among those, we can point out stability, pole location, $H_{2}$ and $H_{\infty}$ specifications that proved to have pure LMI formulations for analysis purpose, as well as for the synthesis of state-feedback [BER 89] or full-order output-feedback controllers, [SCH 97b]. 
SDP: Efficient numerical tools are now available to solve the LMI-based problems. Since the first results of [NES 94] on interior point methods in Semi-Definite Programming (SDP), many improvements were made (see for example [GAH 95], [STU 99] and the report [MIT 01] that gives a comparison between recent softwares). The key features of the existing SDP solvers are first, that they exploit the convexity of the LMI optimisation problem, second, optimality or infeasibility can be attested by duality arguments, third, polynomial time convergence of the algorithms is guaranteed.

Within the chosen framework, all synthesis problems can write as bilinear matrix inequalities (BMIs) and there exist two linearising changes of variables only for the previously cited cases of state-feedback and full-order dynamic output-feedback. No such linearising change of variables exists so far for SOF synthesis and one can conjecture that no convex formulation exists in the general case. Nevertheless, some authors have tackled the SOF problem within the LMI framework through non optimal algorithmic approaches. Focus a particular attention on two recent ones: The first one is a coordinate descent technique where the bilinear terms are made linear by freezing iteratively the different variables (see for example the D-K iteration [ROT 94], iterative LMIs [CAO 98] or also state/output-feedback iterations [PEA 01a]); The second technique applies an elimination argument to transform the BMIs into LMIs constrained by a coupling nonlinear matrix equality $\mathbf{P Q}=\mathbb{1}$. Many algorithms have been proposed to solve this constrained problem such as the alternating projection method [GRI 96], the $X Y$-centring method [IWA 95], the min-max algorithm [GER 95], the cone complementarity linearisation [ELG 97], [LEI 01]. Note that all these quite involved algorithms have some specific convergence properties but lead to local results. In fact, coupled LMIs with the constraint $\mathbf{P Q}=\mathbb{1}$ is proved to be an NP-hard problem [FU 97].

Our goal is not to define new algorithms and we therefore rely on the existing algorithmic techniques that proved their efficiency on many examples [OLI 97]. The contribution holds in a new formulation of the original SOF synthesis problem that proves to have many significant insights for robust multi-objective synthesis.

\section{B. Synthesis by quadratic separation}

The key result of the paper is closely related to topological separation [SAF 80], [GOH 95]. The stability of interconnected systems (figure 1) is tackled via the existence of a topological separator between the graph of the first system $\left(\Sigma_{1}\right)$ and the inverse graph of the other $\left(\Sigma_{2}\right)$.

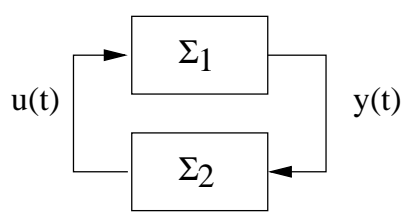

Fig. 1. Interconnected systems

Based on topological separation, major contributions have been made for robust control with respect to uncertainties modelled by a Linear Fractional Transform (LFT). The interconnected systems are the nominal model on one side, and the uncertainties on the other. Robustness is achieved if an operator proves the separation of all inverse graphs representing the admissible uncertainties $\left(\Sigma_{2}=\Delta\right)$ with the unique graph of the nominal system. In [IWA 96], [IWA 98], the topological separation with respect to parametric uncertainty is shown to be achieved by a quadratic separator without any conservatism. In [SCH 97a], [SCH 00], quite similar results are proved to be an extension of the well-known S-procedure [YAK 73]. The NP-hard problem of robust stability with respect to structured uncertainty as defined in the structured singular value $\mu$ problem [SAF 82], [DOY 82] is formulated in [IWA 98] as a quadratic separation problem with an infinite number of constraints. Conservative but finite LMI relaxations are obtained and compared to $(D-G)$ scalings [SCO 98]. Moreover, note that the quadratic separation technique has also been extended from robust stability analysis 
to robust performance analysis such as robust pole location [CHI 99] and robust $H_{2} / H_{\infty}$ guaranteed costs [PEA 00a], [PEA 01b].

While the topological separation proves to be fertile for robustness analysis, at our knowledge, it never was considered for synthesis purpose. The SOF synthesis problem amounts to an interconnected system as in figure 1 where the first system $\Sigma_{1}$ is the given model and $\Sigma_{2}=\mathbf{K}$ is the SOF matrix to compute in order to assess the closed-loop stability. From a separation point of view it is equivalent to find any operator that performs the topological separation between $\Sigma_{1}$ and the inverse graph of some linear transformation. When $\Sigma_{2}=\mathbf{K}$ is a linear transformation, [IWA 98] proves that the separator can be chosen without conservatism among constant quadratic operators. In this framework, the SOF problem is therefore equivalently replaced by the synthesis of some quadratic separator which in turn allows to define an ellipsoidal set of stabilising gains. The major difficulty is to obtain non empty sets.

Roughly speaking, the SOF problem is formulated as LMI conditions for the existence of a quadratic separator defined by three matrices $\mathbf{X}, \mathbf{Y}$ and $\mathbf{Z}$. The condition for the associated set of controllers to be non empty is the non-linear matrix inequality $\mathbf{X} \leq \mathbf{Y Z}^{-1} \mathbf{Y}^{\prime}$. This non-linear constraint is to be regarded form a complexity point of view as equivalent to the constraint PQ $=\mathbb{1}$ of [GRI 96], [IWA 95], [GER 95] (and other).

\section{Multiple specification requirements}

Industrial concern leads to formulate complex requirements for synthesis. The specifications can have multiple aspects such as closed-loop performances and/or robustness. These two aspects are now detailed. Some associated synthesis techniques are cited and the contributions of the quadratic separator-based SOF synthesis are pointed out.

Preformances: The first type of requirements concerns the performances of the closed-loop system. These are typically the stability, the time-response behaviour, the sensitivity to perturbations, the noise attenuation... For most of them, such requirements can be formulated as pole location, $H_{2}$ or $H_{\infty}$ criteria defined on specific models (for each criterion the related model may include weight filters in order to accentuate some frequencies or to shape a time response for example). The synthesis problem is to compute a single control law that achieves all criteria when evaluated on the associated closed-loop models. This synthesis problem is known as multi-objective control. The first results where given in [SCH 97b]. It concerns only statefeedback and full-order output-feedback synthesis and can be applied only in the case when all synthesis models are identical. Additionally, multi-objective synthesis is achieved at the expense of a so called "Lyapunov Shaping Paradigm" that amounts to the conservative choice of a single Lyapunov function for all the criteria. This paradigm was partly relaxed in [APK 00] at the expense of other conservative limitations. An even less conservative relaxation is proposed in [ARZ 02]; The counter part is that it leads to formulate a non-linear constraint on some matrix variables. Finally, it seems that non conservative multi-objective design cannot be performed with LMIs even for the nice cases of state-feedback or full-order output-feedback. The question is then "how many non-linearities need to be introduced in order to solve the SOF multi-objective problem without conservatism?" A major contribution of the chosen quadratic separator-based synthesis is that whatever the number of required performances, the problem has the same structure. Without any restrictive assumption, not even on the system models for each performance specification, the SOF multi-objective problems write as LMIs constrained by the unique non-linear matrix inequality $\mathbf{X} \leq \mathbf{Y Z}^{-1} \mathbf{Y}^{\prime}$. A by-product of this approach has to do with simultaneous stabilisation (see [BLO 94] for an overview) that is proved to be a NP-hard problem [BLO 97]. We reformulate the simultaneous stabilisability as a multi-objective problem with each stability (or performance) requirement defined on a different model.

Robustness: The second requirements have to do with robustness. Due to linearisation simplifications in modelling and to identification limitations, the LTI models are far from being determined with precision. It 
is the seminal consideration leading to the development of the so-called Robust Control theory. In particular, an important feature of the past years was the extension of LMI results to robust control. This was achieved for many uncertainty modellings and at the expense of more or less conservatism. The initial robustness issues based on the quadratic stability concept [BAR 86], [CHI 99], [GER 96], [SCH 97a] have been recently extended to take parameter dependent Lyapunov functions into account, [FER 96], [OLI 99], [PEA 00b], [PEA 01b]. Nevertheless, the robust SOF design suffers form being either highly non-linear or highly conservative. Again, we will show that the quadratic separator-based SOF synthesis is a major contribution with respect to this aspect. In addition, the proposed synthesis technique enables to tackle fragility issues in a new way. This aspect concerns the closed-loop robustness with respect to the presence of uncertainties on the control law parameters. This questioning formulated in [KEE 97] and for which [DOR 98] gives an overview, has significant repercussions for digital controller implementation [D'A 02]. Different techniques have been proposed to deal with fragility issues. Some [YEE 01], [TAK 00], assume that the uncertainties are given while the control law itself has to be designed. Others [YAN 01], give a multiplicative structure to the uncertainty implying that the uncertainty depends on the controller designed values. In all cases, the methodology is quite similar to robustness techniques. The novel approach proposed in the paper is to keep in relation the fragility with the design. The synthesis is performed to design some quadratic separator that defines a whole set of control laws. The system is therefore non-fragile to controller uncertainties as long as the parameters are kept within the designed ellipsoidal set.

\section{Outline}

The multiple contributions and particularities of the quadratic separator design for robust static outputfeedback are exposed in detail in the following outline:

- First, some standard notations are introduced and a useful definition of "matrix ellipsoids" is produced. These ellipsoids are convex sets of matrices defined by some quadratic constraint. Then a result is recalled where the SOF stabilisability is revisited from the quadratic separation point of view. The design of a single stabilising gain is replaced by the synthesis of a matrix ellipsoid.

- The third section is then devoted to fragility. The quadratic separator design for SOF is shown to have important properties with respect to robustness against uncertainties in the control law parameters. The synthesis of non-fragile (resilient) controllers is performed for both additive norm-bounded uncertainty and multiplicative uncertainty.

- Next, the new SOF design is extended to robust synthesis. The quadratic separation approach allows to deal in a similar manner with both the synthesis problem and the robustness issues. The considered uncertainties are rational dissipative.

- In the ensuing section, robust performance is considered. The specifications are either defined by pole location, $\mathrm{H}_{\infty}$ norm or $\mathrm{H}_{2}$ norm. It is shown that these robust performance specifications can be tackled in the same framework as the robust stability.

- The sixth section compounds all results in the general robust multi-objective synthesis. The discussion focuses on the conservatism and the complexity of the SOF design. These remarks conclude the paper, prospective work is detailed.

- The last section gathers all technical proofs. This allows to concentrate, in the main part of the paper, on the issues of the proposed quadratic separator design for robust and resilient static output-feedback. 


\section{PRELIMINARIES}

\section{A. Notations}

Notations are standard.

$\mathbb{R}^{m \times n}$ is the set of $m$-by- $n$ real matrices and $\mathbb{S}^{n}$ is the subset of symmetric matrices in $\mathbb{R}^{n \times n}$. $A^{\prime}$ is the transpose of the matrix $A$ and $A^{*}$ is its transpose conjugate if $A$ is a complex-valued matrix. $A \otimes B$ is the Kronecker product of the matrix $A$ by the matrix $B$.

$\mathbb{1}$ and $\mathbb{O}$ are respectively the identity and the zero matrices of appropriate dimensions.

For symmetric matrices, $>(\geq)$ is the Loëner partial order, i.e., $A>(\geq) B$ if and only if $A-B$ is positive (semi) definite.

Assume $\Sigma_{1}$ and $\Sigma_{2}$ are two systems with appropriate input/output vector dimensions, the interconnected system of figure 1 is denoted $\Sigma_{1} \star \Sigma_{2}$. In case there might be some confusion in the vectors defining the interconnection, the notation is $\Sigma_{1} \stackrel{u, y}{\star} \Sigma_{2}$.

In matrix inequalities as well as in the problem formulations, the decision variables are in bold face.

\section{B. Matrix ellipsoid}

Throughout this paper, a particular set of matrices is used. Before introducing the major results, some properties of this type of sets are described. Due to the notations and by extension of the notion of $\mathbb{R}^{n}$ ellipsoids, these sets are referred to as matrix ellipsoids of $\mathbb{R}^{m \times p}$.

\section{Definition 1:}

Given three matrices $X \in \mathbb{S}^{p}, Y \in \mathbb{R}^{p \times m}$ and $Z \in \mathbb{S}^{m}$, the $\{X, Y, Z\}$-ellipsoid of $\mathbb{R}^{m \times p}$ is the set of matrices $\mathbf{K}$ satisfying the following matrix inequalities:

$$
Z>\mathbb{0} \quad\left[\begin{array}{ll}
\mathbb{1} & \mathbf{K}^{\prime}
\end{array}\right]\left[\begin{array}{cc}
X & Y \\
Y^{\prime} & Z
\end{array}\right]\left[\begin{array}{c}
\mathbb{1} \\
\mathbf{K}
\end{array}\right] \leq \mathbb{0}
$$

By definition, $K_{o} \triangleq-Z^{-1} Y^{\prime}$ is the centre of the ellipsoid and $R \triangleq{ }^{\prime} Z K_{o}-X$ is the radius. The inequalities (1) write also as:

$$
Z>\mathbb{0} \quad\left(\mathbf{K}-K_{o}\right)^{\prime} Z\left(\mathbf{K}-K_{o}\right) \leq R
$$

This definition shows that matrix ellipsoids are special cases of matrix sets defined by a quadratic matrix inequality. It may be possible to define in the same way some hyperbolic or parabolic sets. This paper insists only on ellipsoids. They satisfy the constraint $Z>\mathbb{D}$. Some properties of these sets are pointed out in the following lemma:

\section{Lemma 1:}

i) The $\{X, Y, Z\}$-ellipsoid is non-empty if and only if the radius $(R \geq \mathbb{O})$ is positive semi-definite.

ii) A matrix ellipsoid is a compact convex set.

Proof:

The proof is omitted and can be found in [PEA 02]. 


\section{Definition of SOF stabilisability}

Before getting in the main contributions for robust design, we refer back to some results published in [PEA 02] that cope with static output-feedback (SOF) stabilisability of linear time-invariant (LTI), continuoustime systems.

Consider an LTI system with the state-space representation:

$$
\Sigma:\left\{\begin{array}{l}
\dot{x}(t)=A x(t)+B u(t) \\
y(t)=C x(t)+D u(t)
\end{array}\right.
$$

where $x \in \mathbb{R}^{n}$ is the state vector, $u \in \mathbb{R}^{m}$ is the input control vector and $y \in \mathbb{R}^{p}$ is the output measure vector. A SOF control law is defined by a constant gain matrix $K$, such that:

$$
u(t)=K y(t)
$$

$\Sigma \star K$ is the closed-loop system defined by (3) and (4).

Definition 2:

The LTI system $\Sigma$ is said to be stabilisable by static output-feedback if and only if there exists a matrix gain $\mathbf{K}$ such that $\Sigma \star \mathbf{K}$ is stable.

The stabilisability is tackled in this paper through the use of the Lyapunov theory. The stabilisability of the closed-loop system is attested by the simultaneous search of a quadratic Lyapunov function $V(x)=x^{\prime} \mathbf{P} x$ and of the static feedback gain $\mathbf{K}$ :

\section{Theorem 1:}

The LTI system $\Sigma$ is stabilisable by static output-feedback if and only if there exist two matrices $\mathbf{P} \in \mathbb{S}^{n}$ and $\mathbf{K} \in \mathbb{R}^{m \times p}$ such that:

$$
\left\{\begin{array}{l}
\mathbf{P}>\mathbb{D} \\
\left(A+B \mathbf{K}(\mathbb{1}-D \mathbf{K})^{-1} C\right)^{\prime} \mathbf{P}+\mathbf{P}\left(A+B \mathbf{K}(\mathbb{1}-D \mathbf{K})^{-1} C\right)<\mathbb{0}
\end{array}\right.
$$

Note that the theorem implies to solve non-linear matrix inequalities with respect to the variables $\mathbf{P}$ and K. Perhaps one of the first papers dealing with this problem is [LEV 70] where a non-linear programming approach was proposed. Another well-known necessary and sufficient condition for static output-feedback stabilisability is given in [IWA 94]:

Theorem 2:

The LTI system $\Sigma$ (with $D=\mathbb{0}$ ) is stabilisable by static output-feedback if and only if there exist two matrices $\mathbf{P} \in \mathbb{S}^{n}$ and $\mathbf{Q} \in \mathbb{S}^{n}$ such that:

$$
\begin{cases}\mathbf{P}>\mathbb{0} & C^{\prime} \perp\left(A^{\prime} \mathbf{P}+\mathbf{P} A\right) C^{\perp}<\mathbb{0} \\ \mathbf{Q}>\mathbb{0} & B^{\perp}\left(\mathbf{Q} A^{\prime}+A \mathbf{Q}\right) B^{\prime}<\mathbb{0} \\ \mathbf{P Q}=\mathbb{1} & \end{cases}
$$

where the rows of $B^{\perp}$ and $C^{\perp}$ form a basis for the null space of $B^{\prime}$ and $C^{\prime}$ respectively.

Once a solution to this "dual LMI problem" is found, there exist LMI parametrisations of all static outputfeedback controllers. The difficulty again holds in the non-linearity $\mathbf{P Q}=\mathbb{1}$. In [IWA 95], [GRI 96] different numerical approaches are proposed to address this difficulty.

The following theorem proposes a new quadratic separation-based formulation for the same problem. 


\section{Ellipsoid design for stabilisability}

Theorem 3:

The LTI system $\Sigma$ is stabilisable by static output-feedback if and only if there exist four matrices $\mathbf{P} \in \mathbb{S}^{n}$, $\mathbf{X} \in \mathbb{S}^{p}, \mathbf{Y} \in \mathbb{R}^{p \times m}$ and $\mathbf{Z} \in \mathbb{S}^{m}$ that simultaneously satisfy the following LMI constraints:

$$
\left\{\begin{array}{l}
\mathbf{Z}>\mathbb{0} \\
\mathbf{P}>\mathbb{D} \\
{\left[\begin{array}{ll}
\mathbb{1} & \mathbb{0} \\
A & B
\end{array}\right]^{\prime}\left[\begin{array}{ll}
\mathbb{0} & \mathbf{P} \\
\mathbf{P} & \mathbb{D}
\end{array}\right]\left[\begin{array}{ll}
\mathbb{1} & \mathbb{0} \\
A & B
\end{array}\right]<\left[\begin{array}{cc}
C & D \\
\mathbb{D} & \mathbb{1}
\end{array}\right]^{\prime}\left[\begin{array}{cc}
\mathbf{X} & \mathbf{Y} \\
\mathbf{Y}^{\prime} & \mathbf{Z}
\end{array}\right]\left[\begin{array}{cc}
C & D \\
\mathbb{0} & \mathbb{1}
\end{array}\right]}
\end{array}\right.
$$

and the non-linear inequality constraint:

$$
\mathbf{X} \leq \mathbf{Y Z}^{-1} \mathbf{Y}^{\prime}
$$

Under these conditions a set of stabilising gains $\mathbf{K}$ is given by the non-empty $\{X, Y, Z\}$-ellipsoid:

$$
\left[\begin{array}{ll}
\mathbb{1} & \mathbf{K}^{\prime}
\end{array}\right]\left[\begin{array}{cc}
X & Y \\
Y^{\prime} & Z
\end{array}\right]\left[\begin{array}{c}
\mathbb{1} \\
\mathbf{K}
\end{array}\right] \leq \mathbb{0}
$$

\section{Proof:}

The proof is omitted it can be found in [PEA 02]. It follows the same lines as the proof of theorem 4 given in section VIII.

It is worth noting that theorem 3 is closely related to topological separation. This theory [SAF 80], [GOH 95] applies for the stability analysis of non-linear interconnected systems and has direct connections to the theory of absolute stability. It has had a renewed interest for the past decade in view of robustness analysis where the inter-connexion amounts to an LTI system with an uncertain feedback. Direct consequences of topological separation for robust stability analysis are the IQC (Integral Quadratic Constraint) framework [MEG 97b], [GOH 96], the full-block S-procedure [SCH 00] and the quadratic separation [IWA 98]. In this last paper the stability analysis of interconnected systems where one of the two systems is assumed to be a time-invariant gain is shown to be equivalent to the existence of a matrix called "quadratic separator" subject to some linear matrix constraints. This result highlights the fact that robustness analysis is a convex problem with an infinite number of constraints. Except for some cases where $(D, G)$-scalings are lossless [MEI 97], the robust analysis with respect to structured uncertainty does not have a finite dimensional convex formulation. One main case when the scalings are lossless is when the inter-connexion is composed of an LTI system and a full-block matrix.

While the topological separation proves to be fertile for analysis and in particular for robustness analysis, at our knowledge, it never was considered for synthesis purpose. It is done here for the configuration of figure 2 where $\Sigma(s)=D+C(s \mathbb{1}-A)^{-1} B$ is the system transfer matrix and $\mathbf{K}$ is the full-block matrix control law. The result is a necessary an sufficient condition for closed-loop stability since the scaling is proved to be lossless for this configuration.

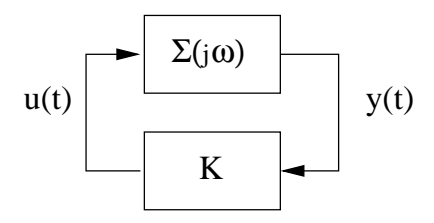

Fig. 2. Interconnected systems for control purpose 


\section{Remark 1:}

The symmetric matrix composed of $\mathbf{X}, \mathbf{Y}$ and $\mathbf{Z}$ is a topological separator between the graph of the system $\Sigma(s)$ and a stabilising set of controllers. One way to demonstrate this is to apply the Kalman-YakubovichPopov lemma, [RAN 96]. The inequality (5) writes as:

$$
\forall \omega \in \mathbb{R} \cup\{\infty\} \quad \mathbb{0}<\left[\begin{array}{c}
\Sigma(j \omega) \\
\mathbb{1}
\end{array}\right]^{*}\left[\begin{array}{cc}
\mathbf{X} & \mathbf{Y} \\
\mathbf{Y}^{\prime} & \mathbf{Z}
\end{array}\right]\left[\begin{array}{c}
\Sigma(j \omega) \\
\mathbb{1}
\end{array}\right]
$$

Inequalities (7) and (8) constitute the separation result. It proves that the quadratic separator simultaneously certifies the stabilisability with respect to a whole set of controllers. The framework adopted in this paper is to design such quadratic separators that define non-empty ellipsoidal sets.

\section{Remark 2:}

The topological separation applies for any linear or non-linear interconnected systems. Therefore the result of theorem 3 is also a sufficient condition for non-linear control. It implies that any control law constrained by the quadratic constraint:

$$
\left(\begin{array}{l}
y(t) \\
u(t)
\end{array}\right)^{\prime}\left[\begin{array}{cc}
\mathbf{X} & \mathbf{Y} \\
\mathbf{Y}^{\prime} & \mathbf{Z}
\end{array}\right]\left(\begin{array}{l}
y(t) \\
u(t)
\end{array}\right) \leq 0
$$

stabilises the system $\Sigma$. The set of stabilising control laws is therefore not limited to the class of static outputfeedback controllers.

In the next sections, the new condition of theorem 3 will be the basis for new developments concerning resilient control and robust multi-objective control.

\section{RESILIENT STABILIS ABILITY}

\section{A. Definitions}

An important insight of theorem 3 concerns the fragility of the control law. By extension of robust stability and quadratic stability, [BAR 85], [GER 91], we define the resilience and the quadratic resilience of a control law:

Definition 3:

Let $K_{o}$ be a stabilising gain for the system $\Sigma$ and let $\Delta_{K}$ be a set of additive uncertainties.

Fragile: The controller is said to be fragile to $\Delta_{K}$ if for some $\Delta_{K} \in \Delta_{K}$ the closed-loop $\Sigma \star K$ with $K=$ $K_{o}+\Delta_{K}$, is unstable.

Resilient: On the contrary, the controller is said to be non-fragile, [YAN 01], or resilient, [COR 99], to $\Delta_{K}$ if the closed-loop is stable for all uncertainties $\Delta_{K} \in \Delta_{K}$.

Quadratically resilient: Moreover, if a unique quadratic Lyapunov function $V(x)=x^{\prime} \mathbf{P} x$ proves the stability of the closed-loop for all uncertainties $\Delta_{K} \in \Delta_{K}$, the system is quadratically resilient.

Resilience and quadratic resilience have the same relationship as robust and quadratic stability. The last two notions concern the properties of the closed-loop system with respect to misknowings on the system model, while resilience deals with uncertainties that may occur when implementing the computed controller model. Resilience and robust stability are generally formulated for parametric uncertainty, i.e. for constant uncertain parameters. On the other hand, quadratic stability and quadratic resilience prove that the stability remains satisfied even for time-varying uncertainty; these notions imply the first ones. 


\section{B. Main fragility results}

\section{Corollary 1:}

Assume the matrices $P, X, Y$ and $Z$ satisfy the constraints (5) and (6). The central controller $K_{o}=-Z^{-1} Y^{\prime}$ is quadratically resilient to all additive uncertainty $\Delta_{K}$ such that:

$$
K=K_{o}+\Delta_{K} \quad \Delta_{K}^{\prime} Z \Delta_{K} \leq R
$$

\section{Proof:}

The proof is straightforward since a single Lyapunov function $V(x)=x^{\prime} P x$ proves the stability of all realisations of the closed-loop (see the proof in [PEA 02]). The fragility issues are a by-product of the quadratic separation as discussed in remark 1.

Corollary 1 is a result for the synthesis of quadratically resilient controllers which belong to a sub-class of all resilient controllers. It is formulated as if the result is only sufficient in the sense that conditions (5) and (6) may exclude some stabilising matrix ellipsoids. It is now proved that the necessity also holds.

\section{Lemma 2:}

The constraints (5) and (6) give a complete parametrisation of all resilient controllers with respect to uncertainties such that (9) with $Z$ positive definite.

\section{Proof:}

Let $K_{o}, Z$ and $R$ be three matrices such that the closed-loop system $\Sigma \star K$ is stable for all gains $K$ such that (9). Define $Y=-K_{o}^{\prime} Z$ and $X=K_{o}^{\prime} Z K_{o}-R$. The statement reads also as "the interconnected system $\Sigma \star K$ is robustly stable with respect to $K$ such that (1)". This amounts to a formulation of a robust stability analysis problem as in [PEA 98a], [XIE 98]. The role of the uncertainty is held by the full-block matrix $K$ for which there is no structural constraint. In this case, it is proved that quadratic stability and robust stability are equivalent and they have an LMI-based formulation:

$$
\exists \boldsymbol{\tau}>0, \exists \mathbf{P}>\mathbb{O}:\left[\begin{array}{cc}
\mathbb{1} & \mathbb{O} \\
A & B
\end{array}\right]^{\prime}\left[\begin{array}{cc}
\mathbb{O} & \mathbf{P} \\
\mathbf{P} & \mathbb{D}
\end{array}\right]\left[\begin{array}{cc}
\mathbb{1} & \mathbb{O} \\
A & B
\end{array}\right]<\boldsymbol{\tau}\left[\begin{array}{cc}
C & D \\
\mathbb{D} & \mathbb{1}
\end{array}\right]^{\prime}\left[\begin{array}{cc}
X & Y \\
Y^{\prime} & Z
\end{array}\right]\left[\begin{array}{cc}
C & D \\
\mathbb{D} & \mathbb{1}
\end{array}\right]
$$

The constraint (5) therefore holds for $P=\frac{1}{\tau} \mathbf{P}$ and (6) holds because $R$ was chosen positive semi-definite.

\section{Some types of control law uncertainties}

The result of corollary 1 illustrates the fact that the ellipsoidal output-feedback design is appropriate to deal with fragility. It gives an admissible set of uncertainties at the end of the design procedure. This set may be characterised by its volume as in [PEA 02]. For example, the volume of the resulting $\{X, Y, Z\}$ ellipsoid could be a reference to compare SOF sets and appreciate their respective resilience. Unfortunately, the volume does not take the geometry into account. Admissible sets can have different geometry properties for an identical volume. When a particular geometry is wanted, the two following corollaries show how to modify the initial matrix inequality problem.

\section{Corollary 2:}

Assume the matrices $P, X, Y$ and $Z$ satisfy (5) with the constraints:

$$
\mathbf{Z}=\mathbb{1} \quad \mathbb{0}<\rho \mathbb{1} \leq \mathbf{Y} \mathbf{Y}^{\prime}-\mathbf{X}
$$

then the central controller $K_{o}=-Y^{\prime}$ is quadratically resilient to all additive norm-bounded uncertainty such that:

$$
K=K_{o}+\Delta_{K} \quad \Delta_{K}^{\prime} \Delta_{K} \leq \rho \mathbb{1}
$$




\section{Proof:}

The proof is due to corollary 1 with the restriction $\mathbf{Z}=\mathbb{1}$ that imposes a spheric geometry for the ellipsoid. Note that the full-block matrix $K$ is not structurally constrained. Therefore, corollary 2 is non-conservative as defined in lemma 2. The resilient design for such norm-bounded additive uncertainties is lossless.

Let the maximally norm-bounded resilient controller (if finite) defined by:

$$
K_{o}^{\mathrm{nb}-\max }=\arg \max \left\{\boldsymbol{\rho}: \forall \Delta_{K}^{\prime} \Delta_{K} \leq \boldsymbol{\rho} \mathbb{1}, \Sigma \star\left(\mathbf{K}_{\mathbf{o}}+\Delta_{K}\right) \text { is stable }\right\}
$$

Due to the losslessness of corollary 2, this maximally resilient controller can be attained by the matrix inequality conditions (5) and (10).

Corollary 3:

Assume the matrices $P, X, Y$ and $Z$ satisfy (5) along with the constraint:

$$
\mathbf{X} \leq\left(1-\bar{\delta}^{2}\right) \mathbf{Y} \mathbf{Z}^{-1} \mathbf{Y}^{\prime}
$$

then the central controller $K_{o}=-Z^{-1} Y^{\prime}$ is quadratically resilient to all multiplicative uncertainty, [COR 99], [YAN 01], such that:

$$
K=K_{o}+\delta K_{o}=(1+\delta) K_{o} \quad|\delta| \leq \bar{\delta}
$$

\section{Proof:}

The proof is direct when writing that condition (2) holds for all $K=K_{o}+\delta K_{o}$ where the absolute value of the scalar $\delta$ is bounded by $\bar{\delta}$. Note that the corollary may be conservative in this case. The proof of lemma 2 is no longer valid due to the structure imposed on $\Delta_{K}=\delta K_{o}$.

Define the maximally multiplicative resilient controller (if finite) by:

$$
K_{o}^{\mathrm{m}-\mathrm{max}}=\arg \max \left\{\overline{\boldsymbol{\delta}}: \forall|\delta| \leq \overline{\boldsymbol{\delta}}, \Sigma \star\left(\mathbf{K}_{\mathbf{o}}+\delta K_{o}\right) \text { is stable }\right\}
$$

Corollary 3 may be conservative and therefore, this maximally resilient controller is possibly not attained by the matrix inequality conditions (5) and (11).

\section{Remark 3:}

The design procedure of theorem 3 needs only to be slightly modified to guarantee resilience with respect to either additive norm-bounded or multiplicative uncertainties. For each type of uncertainty, the non-convex constraint of the initial problem is modified into a quite similar condition. In the sequel, identical remarks are made for all design problems. Quadratic resilience is always assessed; but the non-conservative part, that guarantees the inclusion of all resilient controllers for all admissible control gain uncertainties, tends to fails.

\section{RoBUST STABILISABILITY}

\section{A. Definitions}

Consider the following LTI system:

$$
\Sigma_{\mathrm{lft}}:\left\{\begin{array}{l}
\dot{x}(t)=A x(t)+B_{w} w(t)+B u(t) \\
z(t)=C_{z} x(t)+D_{z w} w(t)+D_{z u} u(t) \\
y(t)=C x(t)+D_{y w} w(t)+D u(t)
\end{array}\right.
$$


The input $w \in \mathbb{R}^{m_{w}}$ and output $z \in \mathbb{R}^{p_{z}}$ define an exogenous feedback of an uncertainty matrix $\Delta$ defined by:

$$
w(t)=\Delta z(t)
$$

For any admissible uncertainty $\Delta$, the uncertain model is an LTI system obtained through the inter-connexion $\Sigma_{\mathrm{lft}}(\Delta)=\Sigma_{\mathrm{lft}} \stackrel{w, z}{\star} \Delta$. The resulting state-space matrices are rational in the uncertain parameters. The interconnexion defines a Linear Fractional Transformation (LFT).

The uncertain parameters are all gathered in a unique matrix $\Delta$. They are assumed to be constant parametric uncertainties and the uncertainty set is a matrix ellipsoid of $\mathbb{R}^{m_{w} \times p_{z}}$ defined by:

$$
\triangle_{\mathrm{lft}}=\left\{X_{\mathrm{lft}}, Y_{\mathrm{lft}}, Z_{\mathrm{lft}}\right\} \text {-ellipsoid }
$$

Such uncertainty sets are also known as $\left\{X_{\mathrm{lft}}, Y_{\mathrm{lft}}, Z_{\mathrm{lft}}\right\}$-dissipative uncertainties. As reported in [MEG 97a], [PEA 98b], [SCO 98], [XIE 98], this modelling of uncertainties contains the well-known norm-bounded uncertainties $(\{-\mathbb{1}, \mathbb{O}, \mathbb{1}\}$-dissipative $)$ and positive real uncertainties $(\{\mathbb{0},-\mathbb{1}, \mathbb{O}\}$-dissipative $)$ which respectively lead to the small gain and passivity frameworks.

In order to guarantee that the nominal system $\Sigma_{\mathrm{lft}}(\mathbb{O})$ is included in the set of realisations $\Sigma_{\mathrm{lft}}(\Delta)$, the matrix $X_{\mathrm{lft}}$ is assumed to be negative semi-definite $\left(X_{\mathrm{lft}} \leq \mathbb{0}\right)$.

Let $\Sigma(\Delta)$ be a generic uncertain model and $\Delta$ any uncertainty set. The general robust stabilisability problem is defined as follows:

\section{Find a gain $\mathbf{K}$ such that the system $\Sigma(\Delta) \star \mathbf{K}$ is stable for all uncertainties $\Delta \in \mathbb{\Delta}$.}

In the assumed case of parametric constant uncertainty, it is acheived by exhibiting for each uncertainty $\Delta \in \Delta$ a parameter-dependent Lyapunov function $V_{r}(x, \Delta)=x^{\prime} \mathbf{P}_{\mathbf{r}}(\Delta) x$ that proves the stability of the closedloop $\Sigma(\Delta) \star \mathbf{K}$.

The quadratic stabilisability problem is defined as follows:

Find a gain $\mathbf{K}$ and a quadratic Lyapunov function $V_{q}(x)=x^{\prime} \mathbf{P}_{\mathbf{q}} x$ such that $V_{q}$ proves the stability of the system $\Sigma(\Delta) \star \mathbf{K}$ for all uncertainties $\Delta \in \mathbb{\Delta}$.

Quadratic stabilisability is a particular instance of robust stabilisability where the Lyapunov matrix is unique over all the set of uncertain parameters $\mathbf{P}_{\mathbf{r}}(\Delta)=\mathbf{P}_{\mathbf{q}}$. To be more precise, quadratic stabilisability is a conservative (sufficient) condition for robust stabilisability. It has nevertheless, major particularities as attested by the considerable and valuable work devoted to this notion.

Robust and quadratic stabilisability are now studied for the case of rational dissipative uncertainty. Quadratic separation is performed simultaneously with respect to the uncertainties $\Delta$ and the control law $\mathbf{K}$.

\section{B. Robust stabilisability results}

\section{Theorem 4:}

The uncertain LTI system $\Sigma_{\mathrm{lft}}(\Delta)$ with $\Delta \in \mathbb{A}_{\mathrm{lft}}$ is robustly stabilisable by static output-feedback if and only if there exist four matrices $\mathbf{P}_{\mathbf{q}} \in \mathbb{S}^{n}, \mathbf{X} \in \mathbb{S}^{p}, \mathbf{Y} \in \mathbb{R}^{p \times m}, \mathbf{Z} \in \mathbb{S}^{m}$ and a scalar $\boldsymbol{\tau}_{\text {lft }}$ that simultaneously satisfy the 
non-linear constraint (6) and the following LMI constraints:

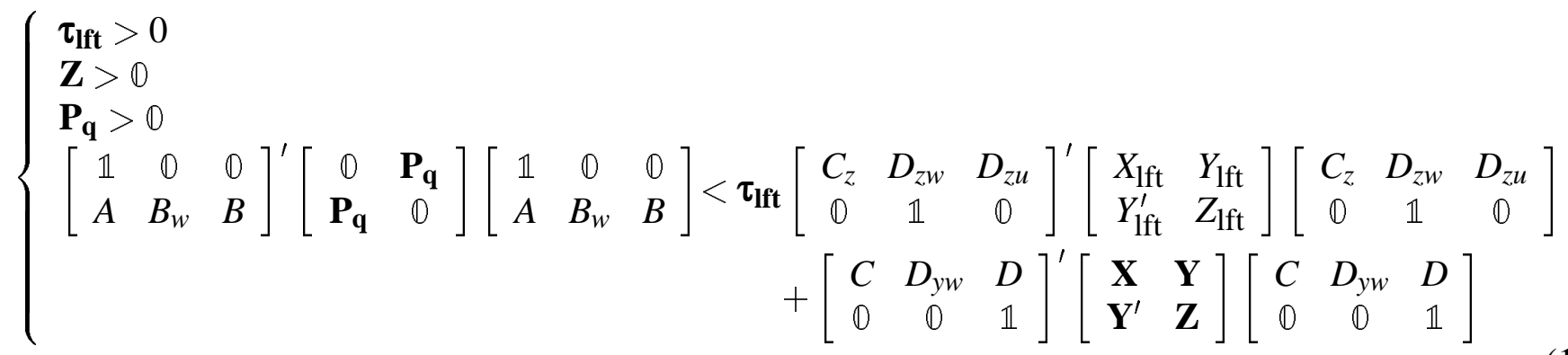

Under these conditions, the $\{X, Y, Z\}$-ellipsoid is a set of quadratically stabilising gains for $\Sigma_{\mathrm{ft}}(\Delta)$ with $\Delta \in \Delta_{\mathrm{lft}}$ and the central controller $K_{o}=-Z^{-1} Y^{\prime}$ is quadratically resilient to all additive uncertainty $\Delta_{K}$ defined by (9).

\section{Proof:}

See the section VIII at the end of the paper to get the complete proof.

Remark 4:

There are close relations between robustness conditions with respect to an exogenous interconnection with some uncertain operator and the proposed $\{X, Y, Z\}$-ellipsoid framework. Both interconnected operators $\Delta$ and $K$ are taken into account using the same theory of quadratic separation (see also remark 1). The first interconnected system $K$ exists if a quadratic separator built out of the matrices $X, Y$ and $Z$ exists. Respectively, robustness is achieved if a quadratic separator exists "between" the uncertainty set and the nominal system. This separation result implies the existence of three matrices $\mathbf{X}_{\Delta}, \mathbf{Y}_{\Delta}$ and $\mathbf{Z}_{\Delta}$ such that:

$$
\begin{array}{rc}
\forall \omega \in \mathbb{R} \cup\{\infty\} & \mathbb{O}<\left[\begin{array}{c}
\Sigma(j \omega, K) \\
\mathbb{1}
\end{array}\right]^{*}\left[\begin{array}{ll}
\mathbf{X}_{\Delta} & \mathbf{Y}_{\Delta} \\
\mathbf{Y}_{\Delta}^{\prime} & \mathbf{Z}_{\Delta}
\end{array}\right]\left[\begin{array}{c}
\Sigma(j \omega, K) \\
\mathbb{1}
\end{array}\right] \\
\forall \Delta \in \Delta & \mathbb{O} \geq\left[\begin{array}{l}
\mathbb{1} \\
\Delta
\end{array}\right]^{*}\left[\begin{array}{ll}
\mathbf{X}_{\Delta} & \mathbf{Y}_{\Delta} \\
\mathbf{Y}_{\Delta}^{\prime} & \mathbf{Z}_{\Delta}
\end{array}\right]\left[\begin{array}{l}
\mathbb{1} \\
\Delta
\end{array}\right]
\end{array}
$$

where $\Sigma(j \omega, K)=\Sigma(j \omega) \stackrel{u, y}{\star} K$ is the transfer matrix of the system seen from the uncertainty operator and where $\Delta$ is any uncertainty set.

The separation result for robustness purpose as discussed in this remark can be also found with more details in [IWA 97], [IWA 98], [PEA 00a] or also in [SCH 97a], [SCH 00] where it is called "full-block Sprocedure" in reference to [YAK 71]. For a complete overview of major results on this subject, the interested reader may also see [IWA 97], [MEI 98], [SCO 98] where various scalings are regarded with respect to the uncertainty description.

In this paper we choose to describe the uncertainty set as a $\left\{X_{\mathrm{lft}}, Y_{\mathrm{lft}}, Z_{\mathrm{lft}}\right\}$-ellipsoid. For these $\left\{X_{\mathrm{lft}}, Y_{\mathrm{lft}}\right.$, $\left.Z_{\mathrm{lft}}\right\}$-dissipative non structured uncertainties, a non conservative scaling exists defined by:

$$
\left[\begin{array}{ll}
\mathbf{X}_{\Delta} & \mathbf{Y}_{\Delta} \\
\mathbf{Y}_{\Delta}^{\prime} & \mathbf{Z}_{\Delta}
\end{array}\right]=\tau_{\mathrm{lft}}\left[\begin{array}{cc}
X_{\mathrm{lft}} & Y_{\mathrm{lft}} \\
Y_{\mathrm{lft}}^{\prime} & Z_{\mathrm{lft}}
\end{array}\right]
$$

It is applied in theorem 4 and enables to assess that all robustly stabilising gains are described (see the necessity part in the proof).

\section{Remark 5:}

Theorem 4 guarantees that all stabilising controllers are described by the conditions (15) and (6) but from a resilience point of view the theorem is conservative. As formulated in lemma 2, the losslessness with respect 
to resilience would be that all robustly stabilising $\{X, Y, Z\}$-ellipsoids are described by the conditions. It would possibly lead to look for maximally resilient control laws. This cannot be done through the formulation of theorem 4 and this is not surprising because $(D, G)$-scaling is conservative for an uncertainty composed of two full-block operators.

Nevertheless, theorem 4 is necessary and sufficient for the synthesis of robust static output-feedback laws in the sense that any robustly stabilising gain belongs to a possibly degenerated (radius $R=\mathbb{O}$ ) ellipsoidal set defined by conditions (15) and (6). Not every robustly stabilising $\{X, Y, Z\}$-ellipsoid is described by the matrix inequality condition, but every robustly stabilising gain belongs to at least one attainable set.

\section{CONTROLLER DESIGN FOR ROBUST PERFORMANCES}

This section is devoted to three examples of robust performances that may be specified for an uncertain LTI system. These are namely the pole location, where the closed-loop poles are specified to lie in some convex region of the complex plane, the $H_{\infty}$ cost, that specifies an over-bounding on the induced $\mathcal{L}_{2}$ norm, and the $H_{2}$ cost, that specifies an over-bounding on the response to white noise or finite energy disturbances. For all these performance specifications, robust synthesis results are given with respect to rational dissipative uncertainty.

\section{A. Robust pole location}

Let a dynamical system be given by its state equation $\dot{x}(t)=A x(t)$ and let $\mathcal{D}$ be a region of the complex plane $\mathbb{C}$. The problem of finding easy testable conditions for a matrix $A$ to have all its roots in the subregion $\mathcal{D}$, i.e. deciding if the dynamical system is $\mathcal{D}$-stable, has been thoroughly investigated in the last years. In [GUT 81], the notion of polynomial regions has been introduced. An extended Lyapunov theorem is then proposed for such regions in [GUT 81], [MAZ 80]. In [CHI 96], and LMI-based characterisation of matrix root-clustering regions is presented. The main result consists in the derivation of an extended Lyapunov matrix equation for a new class of convex subregions named LMI regions and characterised by an LMI in $s$ and $s^{*}$. Here we focus on these LMI regions with a revisited formalism as in [BAC 00], [PEA 00a], [PEA 00b], [HEN 01].

Definition 4:

Let $X_{R}, Y_{R}$ and $Z_{R}$ be square complex valued matrices of same dimensions $\left(\in \mathbb{C}^{d \times d}\right)$ and assume that:

$$
\left[\begin{array}{cc}
X_{R} & Y_{R} \\
Y_{R}^{*} & Z_{R}
\end{array}\right]=\left[\begin{array}{cc}
X_{R} & Y_{R} \\
Y_{R}^{*} & Z_{R}
\end{array}\right]^{*} \quad Z_{R} \geq \mathbb{D}
$$

The $\left\{X_{R}, Y_{R}, Z_{R}\right\}$-region of the complex plane is the convex open set of complex scalars such that:

$$
\left\{s \in \mathbb{C}: X_{R}+s Y_{R}+s^{*} Y_{R}^{*}+s s^{*} Z_{R}<\mathbb{D}\right\}
$$

Examples of the most commonly used $\left\{X_{R}, Y_{R}, Z_{R}\right\}$-regions, [BAC 98], are reminded here. They are all of order $d=1$ and correspond to usual specifications on stability, decay rate or oscillation damping:

- Left-half of the complex plane s.t. $\operatorname{Re}(s)<0$ :

$\{0,1,0\}$-region.

- Left side of a vertical axis s.t. $\operatorname{Re}(s)<\gamma_{r}$ :

- Bottom side of a horizontal axis s.t. $\operatorname{Im}(s)<\gamma_{i}$ :

- Left side of an inclined axis s.t. $\operatorname{Im}(s)<\tan \left(\frac{\pi}{2}-v\right)(\sigma-\operatorname{Re}(s))$ : $\left\{-2 \gamma_{r}, 1,0\right\}$-region. $\left\{-2 \gamma_{i},-j, 0\right\}$-region.

- Unit disk centred at the origin s.t $|s|<1$ :

- Disk centred at $\alpha$ with radius $r$ : $\{2 \sigma \cos (v),(\cos (v)-j \sin (v)), 0\}$-region. $\{-1,0,1\}$-region.
$\left\{\left(\alpha^{2}-r^{2}\right),-\alpha, 1\right\}$-region. 
Regions of order superior to $d=1$ are less frequently used. Regions of order two can be ellipses and parabolas.

In [CHI 96], [CHI 99], the pole location in the intersections of LMI regions is tackled by the augmentation of the order. The intersection of two regions of respective order $d_{1}$ and $d_{2}$ is a region of order $d_{1}+d_{2}$. Here, another approach is adopted. Each region of the intersection is assumed to be a separate objective, i.e. the poles of a system are proved to belong to the intersection if they separately satisfy each pole location objective.

\section{Definition 5:}

An LTI system $\Sigma$ is said to be $\left\{X_{R}, Y_{R}, Z_{R}\right\}$-stable if and only if all its poles belong to the $\left\{X_{R}, Y_{R}, Z_{R}\right\}$-region. An uncertain LTI system is said to be robustly $\left\{X_{R}, Y_{R}, Z_{R}\right\}$-stable if and only if $\Sigma(\Delta)$ is $\left\{X_{R}, Y_{R}, Z_{R}\right\}$-stable for all admissible uncertainty $\Delta \in \mathbb{\Delta}$.

An uncertain LTI system is said to be robustly $\left\{X_{R}, Y_{R}, Z_{R}\right\}$-stabilisable by static output-feedback if there exists a matrix gain $\mathbf{K}$ such that $\Sigma(\Delta) \star \mathbf{K}$ is robustly $\left\{X_{R}, Y_{R}, Z_{R}\right\}$-stable.

\section{Remark 6:}

The definition of $\left\{X_{R}, Y_{R}, Z_{R}\right\}$-stability includes the usual stability of continuous-time $(\dot{x}(t)=A x(t))$ and discrete time systems $(x(t+1)=A x(t))$. In the first case (Hurwitz stability) it amounts to $\{0,1,0\}$-stability while the second case (Schur stability) corresponds to pole location in the $\{-1,0,1\}$-region.

Let the three matrices:

$$
\begin{gathered}
N_{R 1}=\left[\begin{array}{ccc}
\mathbb{1} & \mathbb{0} & \mathbb{0} \\
\mathbb{1}_{d} \otimes A & \mathbb{1}_{d} \otimes B_{w} & \mathbb{1}_{d} \otimes B
\end{array}\right] \quad N_{R 2}=\left[\begin{array}{ccc}
\mathbb{1}_{d} \otimes C_{z} & \mathbb{1}_{d} \otimes D_{z w} & \mathbb{1}_{d} \otimes D_{z u} \\
\mathbb{0} & \mathbb{1} & \mathbb{0}
\end{array}\right] \\
N_{R 3}=\left[\begin{array}{ccc}
\mathbb{1}_{d} \otimes C & \mathbb{1}_{d} \otimes D_{y w} & \mathbb{1}_{d} \otimes D \\
\mathbb{0} & \mathbb{0} & \mathbb{1}
\end{array}\right]
\end{gathered}
$$

\section{Theorem 5:}

If there exist five matrices $\mathbf{P}_{\mathbf{R}} \in \mathbb{S}^{n}, \mathbf{X} \in \mathbb{S}^{p}, \mathbf{Y} \in \mathbb{R}^{p \times m}, \mathbf{Z} \in \mathbb{S}^{m}$ and $\mathbf{T}_{\mathbf{R}} \in \mathbb{S}^{d}$ that simultaneously satisfy the non-linear constraint (6) and the following LMI constraints:

$$
\left\{\begin{array}{l}
\mathbf{T}_{\mathbf{l f t}}>\mathbb{0} \\
\mathbf{Z}>\mathbb{0} \\
\mathbf{P}_{\mathbf{R}}>\mathbb{0} \\
N_{R 1}^{\prime}\left[\begin{array}{cc}
X_{R} \otimes \mathbf{P}_{\mathbf{R}} & Y_{R} \otimes \mathbf{P}_{\mathbf{R}} \\
Y_{R}^{\prime} \otimes \mathbf{P}_{\mathbf{R}} & Z_{R} \otimes \mathbf{P}_{\mathbf{R}}
\end{array}\right] N_{R 1}<N_{R 2}^{\prime}\left[\begin{array}{cc}
\mathbf{T}_{\mathbf{R}} \otimes X_{\mathrm{lft}} & \mathbf{T}_{\mathbf{R}} \otimes Y_{\mathrm{lft}} \\
\mathbf{T}_{\mathbf{R}} \otimes Y_{\mathrm{lft}}^{\prime} & \mathbf{T}_{\mathbf{R}} \otimes Z_{\mathrm{lft}}
\end{array}\right] N_{R 2}+N_{R 3}^{\prime}\left[\begin{array}{cc}
\mathbb{1}_{d} \otimes \mathbf{X} & \mathbb{1}_{d} \otimes \mathbf{Y} \\
\mathbb{1}_{d} \otimes \mathbf{Y}^{\prime} & \mathbb{1}_{d} \otimes \mathbf{Z}
\end{array}\right] N_{R 3}
\end{array}\right.
$$

then the $\{X, Y, Z\}$-ellipsoid is a set of quadratically $\left\{X_{R}, Y_{R}, Z_{R}\right\}$-stabilising gains for $\Sigma_{\mathrm{lft}}(\Delta)$ with $\Delta \in \Delta_{\mathrm{lft}}$ and the central controller $K_{o}=-Z^{-1} Y^{\prime}$ is quadratically resilient to all additive uncertainty $\Delta_{K}$ defined by (9).

Proof:

The proof is omitted. It follows the lines of the proof of theorem 4 and is based on pole location Lyapunov-like results, [CHI 96], [BAC 00], [PEA 00a], [PEA 01b], [PEA 00b], [HEN 01].

\section{B. $H_{\infty}$ control}

A common way of measuring robust performance and disturbance rejection is to use the $\mathcal{L}_{2}$-induced operator norm. The $H_{\infty}$ norm characterises input/output properties in terms of energy to energy, power to power 
and spectrum to spectrum relationships, [ZHO 94]. In this paper the notations are as follows:

$$
\Sigma_{\infty}:\left\{\begin{aligned}
\dot{x}(t) & =A x(t)+B_{w} w(t)+B_{\infty} v_{\infty}(t)+B u(t) \\
z(t) & =C_{z} x(t)+D_{z w} w(t)+D_{z \infty} v_{\infty}(t)+D_{z u} u(t) \\
g_{\infty}(t) & =C_{\infty} x(t)+D_{\infty w} w(t)+D_{\infty} v_{\infty}(t)+D_{\infty u} u(t) \\
y(t) & =C x(t)+D_{y w} w(t)+D_{y_{\infty} v_{\infty}(t)+D u(t)}
\end{aligned}\right.
$$

The input $w$ and the output $z$ define the uncertainty exogenous feedback as in (14). The uncertain system is given by $\Sigma_{\infty}(\Delta)=\Sigma_{\infty} \stackrel{w_{,}, z}{\star} \Delta$. The vector $v_{\infty} \in \mathbb{R}^{m_{\infty}}$ is the input disturbance vector and $g_{\infty} \in \mathbb{R}^{p_{\infty}}$ is the controlled signal. The guaranteed robust $H_{\infty}$ synthesis problem is formulated as follows:

Find a stabilising gain $\mathbf{K}$ such that for all uncertainties the closed-loop transfer from $v_{\infty}$ to $g_{\infty}$ has an $H_{\infty}$ norm less than some specified level $\gamma_{\infty}: \quad \forall \Delta \in \Delta,\left\|\Sigma_{\infty}(\Delta) \stackrel{u, y}{\star} \mathbf{K}\right\|_{\infty}<\gamma_{\infty}$.

Let the four matrices:

$$
\begin{array}{ccc}
N_{\infty 1}=\left[\begin{array}{cccc}
\mathbb{1} & \mathbb{1} & \mathbb{0} & \mathbb{0} \\
A & B_{w} & B_{\infty} & B
\end{array}\right] & N_{\infty 2}=\left[\begin{array}{cccc}
C_{z} & D_{z w} & D_{z \infty} & D_{z u} \\
\mathbb{D} & \mathbb{1} & \mathbb{D} & \mathbb{0}
\end{array}\right] \\
N_{\infty 3}=\left[\begin{array}{cccc}
C_{\infty} & D_{\infty w} & D_{\infty} & D_{\infty u} \\
\mathbb{D} & \mathbb{D} & \mathbb{1} & \mathbb{D}
\end{array}\right] & N_{\infty 4}=\left[\begin{array}{cccc}
C & D_{y w} & D_{y \infty} & D \\
\mathbb{D} & \mathbb{D} & \mathbb{0} & \mathbb{1}
\end{array}\right]
\end{array}
$$

Theorem 6:

If there exist four matrices $\mathbf{P}_{\infty} \in \mathbb{S}^{n}, \mathbf{X} \in \mathbb{S}^{p}, \mathbf{Y} \in \mathbb{R}^{p \times m}, \mathbf{Z} \in \mathbb{S}^{m}$ and two scalars $\tau_{\infty}, \tau_{\text {lfto }}$ that simultaneously satisfy the non-linear constraint (6) and the following LMI constraints:

$$
\left\{\begin{array}{l}
\tau_{\mathrm{lft} \infty}>0 \\
\tau_{\infty}>0 \\
\mathbf{Z}>\mathbb{0} \\
\mathbf{P}_{\infty}>\mathbb{0} \\
N_{\infty 1}^{\prime}\left[\begin{array}{cc}
\mathbb{0} & \mathbf{P}_{\infty} \\
\mathbf{P}_{\infty} & \mathbb{0}
\end{array}\right] N_{\infty 1}<\boldsymbol{\tau}_{\mathrm{lft} \infty} N_{\infty 2}^{\prime}\left[\begin{array}{cc}
X_{\mathrm{lft}} & Y_{\mathrm{lft}} \\
Y_{\mathrm{lft}}^{\prime} & Z_{\mathrm{lft}}
\end{array}\right] N_{\infty 2}+\boldsymbol{\tau}_{\infty} N_{\infty 3}^{\prime}\left[\begin{array}{cc}
-\mathbb{1} & \mathbb{0} \\
\mathbb{0} & \gamma_{\infty}^{2} \mathbb{1}
\end{array}\right] N_{\infty 3}+N_{\infty 4}\left[\begin{array}{cc}
\mathbf{X} & \mathbf{Y} \\
\mathbf{Y}^{\prime} & \mathbf{Z}
\end{array}\right] N_{\infty 4}
\end{array}\right.
$$

then the $\{X, Y, Z\}$-ellipsoid is a set of quadratically stabilising gains such that $\left\|\Sigma_{\infty}(\Delta) \stackrel{u, y}{\star} K\right\|_{\infty}<\gamma_{\infty}$ for all $\Delta \in \Delta_{\mathrm{lft}}$ and the central controller $K_{o}=-Z^{-1} Y^{\prime}$ is quadratically resilient to all additive uncertainty $\Delta_{K}$ defined by (9).

Proof:

The proof may be found in section VIII, at the end of the paper.

\section{C. $\mathrm{H}_{2}$ control}

The input/output performance may also be expressed in terms of $H_{2}$ norm specifications. It allows to characterise wite noise response [PAG 97], [PAG 00] and is frequently used in practical situations. The notations are as follows:

$$
\Sigma_{2}:\left\{\begin{aligned}
\dot{x}(t) & =A x(t)+B_{w} w(t)+B_{2} v_{2}(t)+B u(t) \\
z(t) & =C_{z} x(t)+D_{z w} w(t)+D_{z 2} v_{2}(t)+D_{z u} u(t) \\
g_{2}(t) & =C_{2} x(t)+D_{2 w} w(t)+D_{2} v_{2}(t)+D_{2 u} u(t) \\
y(t) & =C x(t)+D_{y w} w(t)+D_{y 2} v_{2}(t)+D u(t)
\end{aligned} \quad \text { s.t. } \quad\left[\begin{array}{c}
D_{z 2} \\
D_{2} \\
D_{y 2}
\end{array}\right]=\mathbb{D}\right.
$$


The input $w$ and output $z$ define the uncertainty exogenous feedback such as (14) and the uncertain system is given by $\Sigma_{2}(\Delta)=\Sigma_{2}{ }_{\star}^{w, z} \Delta$. The vector $v_{2} \in \mathbb{R}^{m_{2}}$ is the input disturbance vector and $g_{2} \in \mathbb{R}^{p_{2}}$ is the controlled signal. Note that these vectors are assumed to be distinct from the vectors defining the $H_{\infty}$ problem. This allows to separately characterise different performance objectives with various signals. Moreover, in the multi-objective synthesis that is formulated at the end of the section, multiple $H_{\infty}$ and $H_{2}$ performance objectives can be formulated simultaneously.

The guaranteed robust $\mathrm{H}_{2}$ synthesis problem is formulated as follows:

Find a stabilising gain $\mathbf{K}$ such that for all uncertainties the closed-loop transfer from $v_{2}$ to $g_{2}$ has an $\mathrm{H}_{2}$ norm less than some specified level $\gamma_{2}: \quad \forall \Delta \in \Delta,\left\|\Sigma_{2}(\Delta) \stackrel{u, y}{\star} \mathbf{K}\right\|_{2}<\gamma_{2}$.

\section{Remark 7:}

The $H_{2}$ norm of some continuous-time transfer matrix is only defined if the feed-through matrix is zero. For the system (19), the closed-loop feed-through matrix of $\Sigma_{2}(\Delta) \stackrel{u, y}{\star} \mathbf{K}$ is given by:

$$
D_{2}(\Delta, \mathbf{K})=D_{2}+\left[\begin{array}{ll}
D_{2 w} & D_{2 u}
\end{array}\right]\left[\begin{array}{cc}
\Delta & \mathbb{0} \\
\mathbb{0} & \mathbf{K}
\end{array}\right]\left(\mathbb{1}-\left[\begin{array}{cc}
D_{z w} & D_{z u} \\
D_{y w} & D
\end{array}\right]\left[\begin{array}{cc}
\Delta & \mathbb{0} \\
\mathbb{0} & \mathbf{K}
\end{array}\right]\right)^{-1}\left[\begin{array}{c}
D_{z 2} \\
D_{y 2}
\end{array}\right]
$$

Some assumptions are required for $D_{2}(\Delta, \mathbf{K})$ be zero whatever the controller $\mathbf{K}$ and the uncertainty $\Delta$ are. In this paper, the results are given only for the case when $D_{2}, D_{z 2}$ and $D_{y 2}$ matrices are zero. Obviously, other assumptions are possible but they lead to tedious formulations that we chose to avoid here.

Let the four matrices:

$$
\begin{array}{ll}
N_{21}=\left[\begin{array}{lll}
\mathbb{1} & \mathbb{D} & \mathbb{0} \\
A & B_{w} & B
\end{array}\right] & N_{22}=\left[\begin{array}{ccc}
C_{z} & D_{z w} & D_{z u} \\
\mathbb{O} & \mathbb{1} & \mathbb{D}
\end{array}\right] \\
N_{23}=\left[\begin{array}{lll}
C_{2} & D_{2 w} & D_{2 u}
\end{array}\right] & N_{24}=\left[\begin{array}{ccc}
C & D_{y w} & D \\
\mathbb{D} & \mathbb{D} & \mathbb{1}
\end{array}\right]
\end{array}
$$

Theorem 7:

If there exist four matrices $\mathbf{P}_{\mathbf{2}} \in \mathbb{S}^{n}, \mathbf{X} \in \mathbb{S}^{p}, \mathbf{Y} \in \mathbb{R}^{p \times m}, \mathbf{Z} \in \mathbb{S}^{m}$ and two scalars $\boldsymbol{\tau}_{\mathbf{2}}$, $\boldsymbol{\tau}_{\text {lft } 2}$ that simultaneously satisfy the non-linear constraint (6) and the following LMI constraints:

$$
\left\{\begin{array}{l}
\tau_{\mathbf{l f t} 2}>0 \\
\boldsymbol{\tau}_{\mathbf{2}}>0 \\
\mathbf{Z}>\mathbb{0} \\
\mathbf{P}_{\mathbf{2}}>\mathbb{0} \\
\operatorname{Trace}\left(B_{2}^{\prime} \mathbf{P}_{\mathbf{2}} B_{2}\right) \leq \boldsymbol{\tau}_{\mathbf{2}} \gamma_{2}^{2} \\
N_{21}^{\prime}\left[\begin{array}{cc}
\mathbb{0} & \mathbf{P}_{\mathbf{2}} \\
\mathbf{P}_{\mathbf{2}} & \mathbb{0}
\end{array}\right] N_{21}<\tau_{\mathrm{lft} 2} N_{22}^{\prime}\left[\begin{array}{cc}
X_{\mathrm{lft}} & Y_{\mathrm{lft}} \\
Y_{\mathrm{lft}}^{\prime} & Z_{\mathrm{lft}}
\end{array}\right] N_{22}-\tau_{2} N_{23}^{\prime} N_{23}+N_{24}^{\prime}\left[\begin{array}{cc}
\mathbf{X} & \mathbf{Y} \\
\mathbf{Y}^{\prime} & \mathbf{Z}
\end{array}\right] N_{24}
\end{array}\right.
$$

then the $\{X, Y, Z\}$-ellipsoid is a set of quadratically stabilising gains such that $\left\|\Sigma_{2}(\Delta) \stackrel{u, y}{\star} K\right\|_{2}<\gamma_{2}$ for all $\Delta \in \Delta_{\mathrm{lft}}$ and the central controller $K_{o}=-Z^{-1} Y^{\prime}$ is quadratically resilient to all additive uncertainty $\Delta_{K}$ defined by (9).

\section{Proof:}

The proof is put back to section VIII at the end of the paper. 


\section{COMPLEXITY AND CONSERVATISM}

This section is devoted to an evaluation of the ellipsoidal design results in terms of conservatism and complexity. First, using the results on the losslessness of $(D, G)$-scaling, the conservatism of the approach is shown to be directly related to the NP-hard problem of robust stability analysis with respect to structured uncertainty. Next, an extension of the results for multi-objective control shows that a wide class of design problems may be tackled by the approach with the same complexity. At last, some prospectives are drawn in particular in terms of numerical methods.

\section{A. Conservatism}

First, consider the $\mu$ analysis framework for which major results regarding losslessness have been produced.

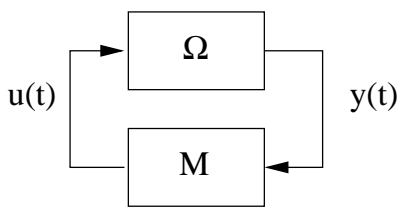

Fig. 3. Interconnection of the $\mu$ framework

Let the interconnected system of figure 3 where $M$ is a given matrix and $\Omega$ a block diagonal uncertain operator such that:

$$
\Omega=\operatorname{diag}\left(\tilde{\omega}_{1} \mathbb{1}, \ldots, \tilde{\omega}_{m_{r}} \mathbb{1}, \omega_{1} \mathbb{1}, \ldots, \omega_{m_{c}} \mathbb{1}, \Omega_{1}, \ldots, \Omega_{m_{F}}\right)
$$

It is composed of $m_{r}$ repeated real scalar blocks, $m_{c}$ repeated complex scalar blocks and $m_{F}$ full-blocks. The $K_{m}$ problem as defined in [SAF 82] is to find the minimal norm over the uncertainty set such that the interconnected system becomes singular. The $\mu$ quantity is defined as the inverse of $K_{m}$, [DOY 82]:

$$
\mu(M)=\frac{1}{\inf \{\|\Omega\|: \mathbb{1}-\Omega M \text { is singular }, \Omega \text { s.t.(21) }\}}
$$

Within this framework the papers [MEI 97], [MEI 98] show that there exist non conservative finite dimensional convex approaches to compute $\mu$ only in the following cases:

- The uncertain operator $\Delta$ is linear time-invariant and $2\left(m_{r}+m_{c}\right)+m_{F} \leq 3$.

- The uncertain operator is linear time-varying.

These convex methods are based on $(D, G)$-scalings when considered in the usual $\mu$ analysis framework. They are shown to be special instances of quadratic separators in [IWA 98] and can also be seen as matrices involved in the full-block S-procedure in [SCH 97a], [SCH 00].

This result concerning the conservatism of the $(D, G)$-scalings is now used to analyse the conservatism of the results proposed in this paper. First, note that $K_{m}$ amounts to the maximal bound on the uncertainty norm $\|\Omega\|$ that keeps the interconnected system non singular. Equivalently, at the expense of an homothecy, it demonstrates the system's stability for all given uncertainties such that $\|\Omega\|^{2}=\Omega^{*} \Omega \leq \mathbb{1}$. Moreover, with tedious manipulations, it can equivalently conclude for the robust stability with respect to uncertainties defined by any quadratic matrix constraint (i.e. $X_{i}+Y_{i} \Omega_{i}+\Omega_{i}^{*} Y_{i}^{*}+\Omega_{i}^{*} Z_{i} \Omega_{i} \leq \mathbb{D}$ ). Such quadratic constraints correspond exactly to the matrix ellipsoids that are used to define the uncertainty set $\Delta_{\mathrm{lft}}$ and the set of controllers.

Now, we take some results from the paper to evaluate if the quadratic separators (i.e. extended $(D, G)$ scalings for any type of quadratically constrained uncertainty) are lossless: 
Case of theorem 3 - The LTI system interconnected with a control law $K$ amounts to an operator $\Omega$ with two blocks $\Omega=\operatorname{diag}(s \mathbb{1}, K)$ where $s$ is the Laplace operator constrained by $s+s^{*} \geq 0$. In this case $m_{r}=0$, $m_{c}=1$ and $m_{F}=1$, the quadratic separator can be chosen without conservatism as the combination of two matrices respectively in the sets:

$$
\Theta_{s}=\left\{\left[\begin{array}{cc}
\mathbb{D} & -\mathbf{P} \\
-\mathbf{P} & \mathbb{D}
\end{array}\right]: \mathbf{P}>\mathbb{O}\right\} \quad, \quad \Theta_{K}=\left\{\left[\begin{array}{cc}
\mathbf{X} & \mathbf{Y} \\
\mathbf{Y}^{\prime} & \mathbf{Z}
\end{array}\right]: \mathbf{X} \leq \mathbf{Y Z}^{-1} \mathbf{Y}^{\prime}\right\}
$$

The lemma 2 about the non-conservatism of the ellipsoidal synthesis with respect to unstructured uncertainty on the control law confirms that the separators are lossless in this case.

Case of corollary 2 - The operator $\Omega$ has the same blocks $\Omega=\operatorname{diag}(s \mathbb{1}, K)$ as in the first case. The only difference is the quadratic constraint on $K$. The quadratic separator can be chosen without conservatism as the combination of a matrix in $\Theta_{s}$ and the other in the set:

$$
\Theta_{\mathrm{bn}-K}=\left\{\left[\begin{array}{cc}
\mathbf{X} & \mathbf{Y} \\
\mathbf{Y}^{\prime} & \mathbb{1}
\end{array}\right]: \mathbb{0}<\rho \mathbb{1} \leq \mathbf{Y} \mathbf{Y}^{\prime}-\mathbf{X}\right\}
$$

Case of corollary 3 - In this case, the operator $\Omega$ can be written as two scalar repeated blocks $\Omega=$ $\operatorname{diag}(s \mathbb{1}, \delta \mathbb{1})$ and $m_{r}=1, m_{c}=1$ and $m_{F}=0$. The quadratic separator is therefore conservative (the operator $\Omega$ is assumed to be time-invariant).

Case of theorem 4 - For the robust stabilisability, the operator $\Omega$ is composed of three blocks, one of which represents the model's uncertainty: $\Omega=\operatorname{diag}(s \mathbb{1}, K, \Delta)$. The uncertainty is assumed to be made of one single full-block. Therefore one gets $m_{r}=0, m_{c}=1$ and $m_{F}=2$ : The quadratic separator is conservative. It is chosen as a combination of matrices in $\Theta_{S}, \Theta_{K}$ and in the set:

$$
\Theta_{\triangle_{\mathrm{lft}}}=\left\{\tau_{\mathrm{lft}}\left[\begin{array}{cc}
X_{\mathrm{lft}} & Y_{\mathrm{lft}} \\
Y_{\mathrm{lft}}^{\prime} & Z_{\mathrm{lft}}
\end{array}\right]: \tau_{\mathrm{lft}}>0\right\}
$$

The conservatism of this case is analysed in remark 5. Theorem 4 is only sufficient for simultaneous robust and resilient control design but losslessness in view of robust (possibly fragile) SOF synthesis.

Case of theorem 5 - In case of robust $\left\{X_{R}, Y_{R}, Z_{R}\right\}$-stabilisability the operator $\Omega$ is highly structured $\Omega=\operatorname{diag}\left(s \mathbb{1}, \mathbb{1}_{d} \otimes K, \mathbb{1}_{d} \otimes \Delta\right)$ and $s$ is constrained in the $\left\{X_{R}, Y_{R}, Z_{R}\right\}$-region. The quadratic separator is chosen as a combination of matrices in the sets:

$$
\begin{gathered}
\Theta_{R}=\left\{\left[\begin{array}{cc}
-Z_{R} \otimes \mathbf{P} & -Y_{R}^{\prime} \otimes \mathbf{P} \\
-Y_{R} \otimes \mathbf{P} & -X_{R} \otimes \mathbf{P}
\end{array}\right]: \mathbf{P}>\mathbb{0}\right\}, \quad \Theta_{\mathbb{1}_{d} \otimes K}=\left\{\left[\begin{array}{cc}
\mathbb{1}_{d} \otimes \mathbf{X} & \mathbb{1}_{d} \otimes \mathbf{Y} \\
\mathbb{1}_{d} \otimes \mathbf{Y}^{\prime} & \mathbb{1}_{d} \otimes \mathbf{Z}
\end{array}\right]: \mathbf{X} \leq \mathbf{Y} \mathbf{Z}^{-1} \mathbf{Y}^{\prime}\right\} \\
\Theta_{\mathbb{1}_{d} \otimes \Delta_{\mathrm{lft}}}=\left\{\left[\begin{array}{cc}
\mathbf{T}_{\mathbf{R}} \otimes X_{\mathrm{lft}} & \mathbf{T}_{\mathbf{R}} \otimes Y_{\mathrm{lft}} \\
\mathbf{T}_{\mathbf{R}} \otimes Y_{\mathrm{lft}}^{\prime} & \mathbf{T}_{\mathbf{R}} \otimes Z_{\mathrm{lft}}
\end{array}\right]: \mathbf{T}_{\mathbf{R}}>\mathbb{D}\right\}
\end{gathered}
$$

The theorem is highly conservative except for regions of order $d=1$. For these regions, one gets that $m_{r}=0$, $m_{c}=1$ and $m_{F}=2$, the same remarks about conservatism can be drawn as for theorem 4 .

Case of theorem 6 - For SOF robust $H_{\infty}$ synthesis the operator $\Omega$ amounts to four blocks such that $\Omega=$ $\operatorname{diag}\left(s \mathbb{1}, K, \Delta, \Delta_{\infty}\right)$ where the last full-block operator $\Delta_{\infty}$ is norm-bounded $\left(\Delta_{\infty}^{*} \Delta_{\infty} \leq \gamma_{\infty}^{2} \mathbb{1}\right)$. This manipulation is equivalent to the classical methods where the $H_{\infty}$ norm of a system is computed for robustness purposes. The theorem is conservative since $m_{r}=0, m_{c}=1$ and $m_{F}=3$. The separators are a combination of matrices in $\Theta_{s}, \Theta_{K}, \Theta_{\triangle_{\mathrm{lft}}}$ and in the set:

$$
\Theta_{\infty}=\left\{\tau_{\infty}\left[\begin{array}{cc}
-\mathbb{1} & \mathbb{0} \\
\mathbb{0} & \gamma_{\infty}^{2} \mathbb{1}
\end{array}\right]: \tau_{\infty}>0\right\}
$$


Note that for systems without uncertainties (the transfer $w \rightarrow z$ is omitted), the $H_{\infty}$ performance synthesis amounts to an operator $\Omega=\operatorname{diag}\left(s \mathbb{1}, K, \Delta_{\infty}\right)$. It can be shown (as in remark 5) that this $H_{\infty}$ performance SOF design is lossless as long as no resiliency constraint is added.

\section{B. Multi-objective synthesis}

Static output-feedback design is in general an open problem. Except for special instances such as statefeedback, no convex finite dimensional formulation exists and one can conjecture that the problem is NP-hard. Some methods used to deal with the SOF synthesis are exposed in the introduction and each of these formulate the synthesis as either a BMI problem or as LMIs constrained by a non-linear equality. With respect to these results, the ellipsoidal synthesis framework has a similar complexity. Moreover, other synthesis problems including resilience, robustness and performance specifications also have a comparable complexity (at the expense of some conservatism as studied in the previous section). It is now shown that all these results can be extended to multi-objective synthesis without increasing the conservatism while preserving the complexity level.

Let the multi-objective problem:

Find a gain $\mathbf{K}$ that simultaneously stabilises various systems with possible robust stability specifications, pole location specifications, input/output performance specifications and resilience constraints.

The multi-objective problem consists in specifying numerous objectives among those defined in the previous sections and in addition, these objectives may be simultaneously solved for various models. Special instances of this general multi-objective problem are:

- Simultaneous stabilisation:

Find a controller $\mathbf{K}$ that simultaneously stabilises a finite number of systems.

Such requirements tackle the "integrity" of systems (stability is expected whatever some discrete perturbations such as failure of components or structural changes may be) and form also a non-guaranteed but sometimes efficient approach for uncertain or non-linear systems (stability is expected for some extreme values of the uncertainty or for some linearised models around operating points). See [BLO 95] for an overview.

- $H_{2} / H_{\infty}$ control:

Find a controller $\mathbf{K}$ that satisfies both a $\mathrm{H}_{2}$ and a $H_{\infty}$ constraint on the closed-loop system.

This most simple multi-objective problem is perhaps the most famous one because it enables to simultaneously give a robust stability requirement ( $H_{\infty}$ constraint) and specifications on transient responses $\left(H_{2}\right.$ objective).

- Robust pole location in the intersection of LMI regions:

Find a controller $\mathbf{K}$ such that for any parametric uncertainty, the closed-loop poles belong to the specified intersection of regions.

Such specification allows for example to simultaneously constrain the decay rate (region limited by a vertical axis), the damping ratio (region limited by a sector) and the undamped natural frequency (region limited by a disc centred at the origin). When dealing with LTI systems without uncertainties, such specifications can be guaranteed with a single quadratic Lyapunov matrix over all regions [CHI 96]. This is no longer the case for robust pole location. In order to avoid conservatism, each region must be treated separately involving a specific Lyapunov function.

Without getting into details, it is quite clear that for practical purposes the multi-objective problems are the most adapted. When dealing with a concrete system, the aim is quite often to be able to simultaneously 
specify some concrete requirements such as noise attenuation, perturbation rejection, time responses, loop shaping with weight functions, robustness... When the design procedure fails, it is possible to relax one or more specifications and conclude about the influence of each requirement. Although the multi-objective problem has such an importance in practice, very few theoretical methods allow to come close to such a general formulation. Among these, the major result is the one relying on the "Lyapunov Shaping Paradigm" in [SCH 97b]. It shows how to solve multi-objective problems in the case of full-order output-feedback and can be applied only when the model is unique. Moreover, it introduces an extra conservatism by the use of a single Lyapunov matrix for all objectives.

In the $\{X, Y, Z\}$-ellipsoid framework exposed in this paper, the technique for dealing with multi-objective specifications is in a sense inherited of [SCH 97b]. Each objective is taken into account by gathering together all related LMI conditions:

For each model and each objective, find the matrix unknowns (Lyapunov matrices, $\mathbf{P}$, scalars $\pi$, scalings $\left.\mathbf{T}_{\mathbf{R}} \ldots\right)$ plus the three common matrices $\mathbf{X}, \mathbf{Y}$ and $\mathbf{Z}$, constrained by all the concatenated LMI constraints and the unique non-linear constraint (6).

In other words, to solve a multi-objective problem, we need only to increase the number of unknowns and the number of LMI constraints accordingly to each objective and each model. From a numerical point of view, it increases the computation burden and reduces the domain where the $\{X, Y, Z\}$-ellipsoid can be found. From a theoretical point of view, there is no additional conservatism to solve a multi-objective control problem compared to single-objective control problems. Moreover, as in a single-objective control problem, all the constraints are convex (LMI) except for the unique non-linear constraint which can be either (6), (10) or (11) depending on the expected resilience of the designed control law.

\section{Numerical methods}

Consider that there is no resilience specification (if any, the same remarks will identically hold with the modified non-linear constraint). All design problems are equivalent to finding a feasible solution $(\mathbf{Q}, \mathbf{X}, \mathbf{Y}, \mathbf{Z})$ to the constraints summarised as:

$$
\mathcal{L}(\mathbf{Q}, \mathbf{X}, \mathbf{Y}, \mathbf{Z})<\mathbb{0} \quad \text { and } \quad \mathbf{X} \leq \mathbf{Y Z}^{-1} \mathbf{Y}^{\prime}
$$

where $\mathbf{Q}$ represents all the stacked variables such as the Lyapunov matrices and other separators, and where $\mathcal{L}(\cdot)$ is a linear matrix operator. The first constraint $\mathcal{L}(\mathbf{Q}, \mathbf{X}, \mathbf{Y}, \mathbf{Z})<\mathbb{0}$ is convex and there exist efficient numerical tools to solve such LMI constraints. The main difficulty comes from the non-linear constraint.

A common technique to tackle the non-linearity is to transform the feasibility problem into an optimisation problem such as:

$$
q^{*}=\min _{\hat{L}(\hat{\mathbf{Q}})<0} f(\hat{\mathbf{Q}})
$$

where $\mathbf{Q}$ represents all variables (some relaxations possibly lead to additional variables), where $f(\cdot)$ is a nonlinear scalar function and $\hat{\mathcal{L}}$ is a linear matrix operator. The transformation has to be lossless in the following sense: "There exists a feasible point to (22) if and only if $q^{*} \leq 0 . "$

First-order suboptimal algorithms can be applied to this optimisation problem. Starting from an initial acceptable point $\hat{\mathcal{L}}\left(\hat{Q}_{0}\right)<\mathbb{O}$, a linear approximation of the non-linear objective is iteratively minimised:

$$
\text { step k : } \quad \hat{Q}_{k+1}=\arg \min _{\hat{L}(\hat{\mathbf{Q}})<0}<\nabla f\left(\hat{Q}_{k}\right) \mid \hat{\mathbf{Q}}>
$$


Each step is an LMI problem and can be solved efficiently using the existing semi-definite solvers [GAH 95], [STU 99]. The algorithm succeeds as soon as the objective becomes equal to zero. Unfortunately, no conclusion can be drawn if the convergence stops (slow evolution of the criterion) at a positive value of the criterion.

Among possible optimisation formulations, two are pointed out:

- If the control law has a scalar input $(p=1)$, then the non-linear constraint is scalar and one can take $f(\hat{\mathbf{Q}})=\mathbf{X}-\mathbf{Y Z}^{-1} \mathbf{Y}^{\prime} \in \mathbb{R}$. This particular case concerns the control design for MISO (multi-input, singleoutput) systems.

- For any MIMO (multi-input, multi-output) system, perform a cone complementarity technique that leads to a criterion of the type $f(\hat{\mathbf{Q}})=$ trace $(\mathbf{M N})$. This method has been applied in [PEA 02] and showed to be highly efficient. In particular, when tested on low dimensional examples it appeared that the algorithm always converge.

These two formulations show that there is no unique way to build the optimisation problem (23). Moreover, the dimension $p$ of the non-linear constraint may condition the efficiency of the first-order algorithm. Intensive experimentations should be performed to draw valuable conclusions and we believe that dedicated optimisation algorithms may emerge. Prospective work will focus on these numerical aspects, going further than the first encouraging results in [PEA 02].

\section{CONCLUSIONS}

The paper exposes a novel approach for control design. The output-feedback control is viewed with respect to topological separation theory so that the computation of a single control law is replaced by the design of a quadratic separator. The new formulation is lossless and has the major benefit for decoupling the controller computation from closed-loop specifications such as robustness or input/output performance.

All results are given with respect to the design of static output-feedback controllers for linear timeinvariant, continuous-time systems. The tackled problems are the fragility of the control law, the robustness with respect to rational dissipative uncertainty, the robust pole location, the robust $H_{\infty}$ control and the robust $\mathrm{H}_{2}$ control. In turn, all these problems can be aggregated together without conservatism to form the general resilient, robust multi-objective problem. A wide range of automatic control problems fall within this category and they are shown to have common complexity.

From a computational point of view, every considered controller design is achieved by the solutions to a non-linear matrix inequality $\mathbf{X} \leq \mathbf{Y} \mathbf{Z}^{-1} \mathbf{Y}^{\prime}$ constrained by purely linear matrix inequalities. As for other existing static output-feedback results, the new formulation is NP-hard. Some suggestions for adapted algorithms based on semi-definite programming are driven.

\section{REFERENCES}

[APK 00] P. Apkarian, P. Pellanda and H. Tuan, "Mixed $H_{2} / H_{\infty}$ Multi-Channel Linear Parameter-Varying Control in Discrete Time", proceedings of American Control Conference, Chicago, Il, june 2000, pages 1322-1326.

[ARZ 00] D. ARZELIER and D. PEAUCELle, "Robust Multi-Objective State-Feedback Control for Real Parametric Uncertainties via ParameterDependent Lyapunov Functions", proceedings of ROCOND, Prague, june 2000.

[ARZ 02] D. Arzelier, D. Henrion and D. Peaucelle, "Robust D-stabilization of a Polytope of Matrices", Int. J. Control, vol. 75, no. 10, 2002, pages 744-752.

[BAC 98] O. BACHELIER, "Commande des Systèmes Linéaires Incertains Placement de Pôles Robuste en $\mathcal{D}$-Stabilité", PhD thesis, Institut Nathional des Sciences Appliquées de Toulouse, 1998.

[BAC 00] O. BAChelier, D. Peaucelle, D. Arzelier and J. Bernussou, "A Precise Robust Matrix Root-clustering Analysis with Respect to Polytopic Uncertainty", proceedings of American Control Conference, Chicago Illinois, june 2000, pages 3331-3335.

[BAR 85] B. BARMISH, "Necessary and Sufficient Conditiond for Quadratic Stabilizability of an Uncertain System", J. Optimization Theory and Applications, vol. 46, no. 4, 1985. 
[BAR 86] B. BARMish and C. DeMarco, "A New Method for Improvement of Robustness Bounds for Linear State Equations", proceedings of Princeton Conf. Inform. Sci. Syst, 1986.

[BER 89] J. Bernussou, J. Geromel and P. Peres, "A Linear Programing Oriented Procedure for Quadratic Stabilization of Uncertain Systems", Systems \& Control Letters, vol. 13, 1989, pages 65-72.

[BER 92] D. BERnSTEIn, "Some Open Problems in Matrix Theory Arising in Linear Systems and Control”, Linear Algebra Applications, vol. 162-164, 1992, pages 409-432.

[BLO 94] V. BLONDEL, Simultaneous Stabilization of Linear Systems, Springler-Verlag, London, 1994.

[BLO 95] V. Blondel, M. Gevers and A. Lindquist, "Survey on the State of Systems and Control", European J. of Control, vol. 1, 1995, pages 5-23.

[BLO 97] V. BLONDE and J. TsitsiKLIS, "NP-hardness of some linear control design problems", SIAM J. Control and Optimization, vol. 35, no. 6, 1997, pages 2118-2127.

[BOY 94] S. BOYD, L. E. GHAOUi, E. Feron and V. BALAKRISHnAn, Linear Matrix Inequalities in System and Control Theory, SIAM Studies in Applied Mathematics, Philadelphia, 1994.

[CAO 98] Y.-Y. CAO and Y.-X. SUN, "Static Output Feedback Simultaneous Stabilization: ILMI Approach", Int. J. Control, vol. 70, no. 5, 1998, pages 803-814.

[CHI 96] M. ChILALI and P. GAhInet, "H$H_{\infty}$ design with pole placement constraints: An LMI approach", IEEE Trans. on Automat. Control, vol. 41, 1996, pages 358-367.

[CHI 99] M. Chilali, P. Gahinet and P. ApKarian, "Robust Pole Placement in LMI Regions", IEEE Trans. on Automat. Control, vol. 44, no. 12,1999 , pages $2257-2270$.

[COR 99] J. CORRADO and W. HADDAD, "Static Output Feedback Controllers for Systems with Parametric Uncertainty and Controller Gain Variations", proceedings of American Control Conference, San Diego, june 1999, pages 915-919.

[D'A 02] R. D'ANDREA and R. IstePANIAN, "Design of full state feedback finite-precision controllers", Int. J. of Robust and Nonlinear Control, vol. 12, 2002, pages 537-553.

[DOR 98] P. DORATo, "Non-fragile Controller Design: an Overview", proceedings of American Control Conference, Philadelphia, Pennsylvania, june 1998, pages 2829-2831.

[DOY 82] J. DOYLE, "Analysis of feedback systems with structured uncertainties", IEEE Trans. on Automat. Control, vol. 129, no. 6, 1982, pages $255-267$.

[DOY 94] J. Doyle, K. Zhou, K. Glover and B. Bodenheimer, "Mixed $H_{2}$ and $H_{\infty}$ Performance Objectives II: Optimal Control", IEEE Trans. on Automat. Control, vol. 39, no. 8, 1994, pages 1575-1586.

[ELG 97] L. El Ghaoui, F. Oustry and M. AitRami, "A Cone Complementarity Linearization Algorithm for Static Ouput-Feedback and Related Problems", IEEE Trans. on Automat. Control, vol. 42, no. 8, 1997, pages 1171-1176.

[ELG 00] L. El GhaOUI and S.-I. NiCUleSCU, editors, Advances in Linear Matrix Inequality Methods in Control, Advances in Design and Control, SIAM, Philadelphia, 2000.

[FER 96] E. Feron, P. ApKarian and P. Gahinet, "Analysis and Synthesis of Robust Control Systems via Parameter-Dependent Lyapunov Functions", IEEE Trans. on Automat. Control, vol. 41, no. 7, 1996, pages 1041-1046.

[FU 97] M. FU and Z.-Q. LUO, "Computational Complexity of a Problem Arising in Fixed Order Output Feedback Design", Systems \& Control Letters, vol. 30, 1997, pages 209-215.

[GAH 95] P. Gahinet, A. Nemirovski, A. Laub and M. Chilali, LMI Control Toolbox User's Guide, The Mathworks Partner Series, 1995.

[GER 91] J. Geromel, P. Peres and J. Bernussou, "On a Convex Parameter Space Method for Linear Control Design of Uncertain Systems", SIAM J. Control and Optimization, vol. 29, no. 2, 1991, pages 381-402.

[GER 95] J. Geromel, DE C. SouzA and R. Skelton, "LMI Numerical Solution for Output Feedback Stabilisation", proceedings of Conference on Decision and Control, New Orleans, LA, december 1995, pages 40-44.

[GER 96] J. Geromel, P. Peres and S. SouzA, "Convex Analysis of Output Feedback Control Problems: Robust Stability and Performance", IEEE Trans. on Automat. Control, vol. 41, no. 7, 1996, pages 997-1003.

[GOH 95] K. GOH and M. SAFOnOv, "Robust Analysis, Sector and Quadratic Functionals", proceedings of Conference on Decision and Control, New Orleans, LA, december 1995.

[GOH 96] K. GoH, "Structure and Factorization of Quadratic Constraints for Robustness Analysis", proceedings of Conference on Decision and Control, Kobe, Japan, december 1996, pages 4649-4654.

[GRI 96] K. GRIGORIADIS and R. Skelton, "Low Order Design for LMI Problems Using Alternating Projection Methods", Automatica, vol. 32 , no. 8, 1996, pages 1117-1125.

[GUT 81] S. GUTMAN and E. JURY, "A general theory for matrix root-clustering in subregions of the complex plane", IEEE Trans. on Automat. Control, vol. 26, no. 4, 1981, pages 853-863.

[Hen 01] D. Henrion, D. Arzelier, D. Peaucelle and M. Sebek, "An LMi Condition for Robust Stability of Polynomial Matrix Polytopes", Automatica, vol. 37, no. 3, 2001, pages 461-468.

[IWA 94] T. IWASAKI and R. SkELTON, "All Controllers for the General $H_{\infty}$ Control Problem. LMI Existence Conditions and State Space Formulas", Automatica, vol. 30, no. 8, 1994, pages 1307-1317.

[IWA 95] T. IWASAKI and R. SKELTON, "The XY-centring Algorithm for the Dual LMI Problem: a new approach to fixed-order control design", Int. J. Control, vol. 62, no. 6, 1995, pages 1257-1272.

[IWA 96] T. IWASAKI and S. HARA, "Well-Posedness of Feedback Systems: insights into exact robustness analysis", proceedings of Conference on Decision and Control, Kobe, Japan, december 1996, pages 1863-1868.

[IWA 97] T. IWASAKI, "Robust stability analysis with quadratic separator: Parametric time-varing uncertainty case", proceedings of Conference on Decision and Control, San Diego, CA, december 1997.

[IWA 98] T. IWASAKI and S. HARA, "Well-Posedness of Feedback Systems: Insights into Exact Robustness Analysis and Approximate Computations", IEEE Trans. on Automat. Control, vol. 43, no. 5, 1998, pages 619-630.

[KEE 97] L. KeEL and S. Bhattacharyya, "Robust, Fragile, or Optimal?", IEEE Trans. on Automat. Control, vol. 42, no. 8, 1997, pages 1098-1105.

[LEI 01] F. LEIBFRITZ, “An LMI-based algorithm for designing suboptimal static $H_{2} / H_{\infty}$ output feedback controllers.”, SIAM Journal on Control and Optimization, vol. 39, no. 6, 2001, pages 1711 - 1735. 
[LEV 70] W. Levine and M. Athans, "On the Determination of the Optimal Constant Output Feedback Gains for Linear Multivariable Systems", IEEE Trans. on Automat. Control, vol. 15, no. 1, 1970.

[MAZ 80] A. MAZCO, "The Lyapunov matrix equation for a certain class of regions bounded by algebraic curves", Soviet. Automatic Control, 1980.

[MEG 97a] A. Megreski and A. RANTZER, "System Analysis via Integral Quadratic Constraints", IEEE Trans. on Automat. Control, vol. 42, no. 6,1997 , pages $819-830$.

[MEG 97b] A. MEgretski and A. Rantzer, "System Analysis via Integral Quadratic Constraints", IEEE Trans. on Automat. Control, vol. 42, no. 6, 1997, pages 819-830.

[MEI 97] G. Meinsma, Y. Shrivastava and M. FU, "A dual formulation of mixed $\mu$ and on the losslessness of $(D, G)$-scaling", IEEE Trans. on Automat. Control, vol. 42, no. 7, 1997, pages 1032-1036.

[MEI 98] G. MeINSMa, T. IWASAKI and M. FU, "On stability robustness with respect to LTV uncertainties", proceedings of Conference on Decision and Control, Tampa, Florida USA, december 1998, pages 4408-4409.

[MIT 01] H. MitTELmanN, “An Independent Benchmarking of SDP and SOCP Solvers", Tech. report, july 2001, Arizona State University, URL: www.optimization-online.org/DB_HTML/2001/07/358.html.

[NES 94] Y. NESTEROV and A. NemIROVSKII, Interior-Point Polynomial Algorithms in Convex Programming, SIAM Studies in Applied Mathematics, Philadelphia, PA, 1994.

[OLI 97] DE M. OliVEIRA and J. Geromel, "Numerical Comparison of Output Feedback Design Methods", proceedings of American Control Conference, Albuquerque, New Mexico, june 1997.

[OLI 99] De M. Oliveira, J. Bernussou and J. Geromel, “A New Discrete-Time Stability Condition”, Systems \& Control Letters, vol. 37, no. 4, 1999, pages 261-265.

[PAG 97] F. PAGANini and E. Feron, "Analysis of Robust $\mathrm{H}_{2}$ Performance: Comparison and Examples", proceedings of Conference on Decision and Control, San Diego, CA, USA, december 1997, pages 1000-1005.

[PAG 00] F. PAGANINI and E. FERON, "Advances in Linear Matrix Inequality Methods in Control", Chapter 7 Linear Matrix Inequality Methods for Robust $\mathrm{H}_{2}$ Analysis: A Survey with Comparisons, pages 129-150, Advances in Design and Control, SIAM, 2000, edited by L. El Ghaoui and S.-I. Niculescu.

[PEA 98a] D. PeAuCElle and D. Arzelier, "Robust Disk Pole Assignment by State and Dynamic Output Feedback for Generalised Uncertainty Models - An LMI Approach", proceedings of Conference on Decision and Control, Tampa, Fl, USA, december 1998, pages 1728-1733.

[PEA 98b] D. Peaucelle, D. ArZelier and G.GARCIA, "Quadratic Stabilisability and Disk Pole Assignment for Generalised Uncertainty Models - An LMI Approach", proceedings of 2nd IMACS multiconference CESA, vol. 1, april 1998, pages 650-655.

[PEA 00a] D. PEAUCELle, "Formulation Générique de Problèmes en Analyse et Commande Robuste par les Fonctions de Lyapunov Dépendant des Paramètres", PhD thesis, Université Toulouse III - Paul Sabatier, France, july 2000.

[PEA 00b] D. Peaucelle, D. Arzelier, O. Bachelier and J. Bernussou, "A New Robust D-Stability Condition for Real Convex Polytopic Uncertainty", Systems \& Control Letters, vol. 40, no. 1, 2000, pages 21-30.

[PEA 01a] D. PeAucelle and D. Arzelier, "An Efficient Numerical Solution for $\mathrm{H}_{2}$ Static Output Feedback Synthesis", proceedings of European Control Conference, Porto, Portugal, september 2001, pages 3800-3805.

[PEA 01b] D. Peaucelle and D. Arzelier, "Robust Performance Analysis with LMI-Based Methods for Real Parametric Uncertainty via Parameter-Dependent Lyapunov Functions", IEEE Trans. on Automat. Control, vol. 46, no. 4, 2001, pages 624-630.

[PEA 02] D. PeAucelle, D. Arzelier and R. Bertrand, "Ellipsoidal Sets for Static Output Feedback", proceedings of 15th IFAC World Congress, Barcelona, july 2002.

[RAN 96] A. RANTZER, “On the Kalman-Yakubovitch-Popov Lemma”, Systems \& Control Letters, vol. 28, 1996, pages 7-10.

[ROT 94] M. ROTEA and T. IWASAKI, "An alternative to the D-K iteration?", proceedings of American Control Conference, Baltimore, june 1994, pages 53-57.

[SAF 80] M. SAFOnov, Stability and Robustness of Multivariable Feedback Systems, Signal Processing, Optimization, and Control, MIT Press, 1980.

[SAF 82] M. SAFOnOv, "Stability Margins of Diagonnaly Perturbed Multivariable Feedback Systems", proceedings of IEE Proceedings, vol. 129, 1982, pages 251-256.

[SCH 97a] C. SCHERER, "A Full Block S-Procedure with Applications", proceedings of Conference on Decision and Control, San Diego, CA, december 1997, pages 2602-2607.

[SCH 97b] C. Scherer, P. Gahinet and M. Chilali, "Multiobjective Output-Feedback Control via LMI optimisation", IEEE Trans. on Automat. Control, vol. 42, no. 7, 1997, pages 896-911.

[SCH 00] C. SCHERER, "Advances in Linear Matrix Inequality Methods in Control", Chapter 10 Robust Mixed Control and Linear ParameterVarying Control with Full Block Scallings, pages 187-207, Advances in Design and Control, SIAM, 2000, edited by L. El Ghaoui and S.-I. Niculescu.

[SCO 98] G. SCORLETTI and L. E. GhaOuI, "Improved LMI Conditions for Gain Scheduling and Related Control Problems", Int. J. of Robust and Nonlinear Control, vol. 8, 1998, pages 845-877.

[SKE 98] R. Skelton, T. IwAZAKI and K. GRIgORIADIs, A unified Approach to Linear Control Design, Taylor and Francis series in Systems and Control, 1998.

[STU 99] J. STURM, "Using SeDuMi 1.02, a MATLAB toolbox for optimization over symmetric cones", Optimization Methods and Software, vol. 11-12, 1999, pages 625-653, URL: fewcal. kub.nl /sturm/software/sedumi.html.

[SYR 97] V. Syrmos, C. Abdallah, P. Dorato and K. Grigoriadis, "Static Output Feedback: A Survey", Automatica, vol. 33, no. 2, 1997, pages 125-137.

[TAK 00] R. Takahashi, D. Dutra, R. Palhares and P. Peres, "On Robust Non-Fragile Static State-Feedback Controller Synthesis", proceedings of Conference on Decision and Control, Sydney, Autralia, december 2000, pages 4909-4914.

[TUA 00] H. TUan, P. ApKARIAn and T. NGUYEN, "Robust and Reduced-order Filtering: New Characterizations and Methods", proceedings of American Control Conference, Chicago, Il, june 2000, pages 1327-1331.

[XIE 98] S. XIE, L. XIE and C. DE SoUZA, "Robust Dissipative Control for Linear Systems with Dissipative Uncertainty", Int. J. Control, vol. 70, no. 2, 1998, pages 169-191.

[YAK 71] V. YAKUBOVITCH, “The S-procedure in nonlinear control theory”, Vestnik Leningrad University, , 1971, pages 62-77. 
[YAK 73] V. A. YAKUBOVICH, "Minimization of quadratic functionals under the quadratic constraints and the necessity of a frequency condition in the quadratic criterion for absolute stability of nonlinear control systems", Soviet Math. Doklady, vol. 14, 1973, pages 593-597.

[YAN 01] G.-H. YANG and J. WANG, "Non-fragile $H_{\infty}$ control for linear systems with multiplicative controller gain variations", Automatica, vol. 37, 2001, pages 727-737.

[YEE 01] J.-S. YEE, G.-H. YANG and J. WANG, "Non-fragile guaranteed cost control for discrete-time uncertain linear systems", Int. J. Systems Science, vol. 32, no. 7, 2001, pages 845-853.

[ZHO 94] K. Zhou, K. Glover, B. Bodenheimer and J. Doyle, "Mixed $H_{2}$ and $H_{\infty}$ Performance Objectives I: Robust Performance Analysis", IEEE Trans. on Automat. Control, vol. 39, no. 8, 1994, pages 1564-1574.

\section{PROOFS}

\section{A. Proof of sufficiency in theorem 4}

Assume conditions (15) and (6) hold for some choice of $P_{q}, X, Y, Z$ and $\tau_{\mathrm{lft}}$. Take any matrix $K$ such that (9). It belongs to the $\{X, Y, Z\}$-ellipsoid defined by (1). The ellipsoid is non empty due to condition (6) and lemma 1. The Finsler's lemma [SKE 98] applied to condition (1) implies that there exists a strictly positive scalar $\tau>0$ such that:

$$
\left[\begin{array}{ll}
X & Y \\
Y^{\prime} & Z
\end{array}\right] \leq \tau\left[\begin{array}{c}
K^{\prime} \\
-\mathbb{1}
\end{array}\right]\left[\begin{array}{ll}
K & -\mathbb{1}
\end{array}\right]
$$

For this choice of $K$ and $\tau$ one gets:

$$
\begin{aligned}
{\left[\begin{array}{ccc}
\mathbb{1} & \mathbb{D} & \mathbb{0} \\
A & B_{w} & B
\end{array}\right]^{\prime}\left[\begin{array}{cc}
\mathbb{0} & P_{q} \\
P_{q} & \mathbb{0}
\end{array}\right]\left[\begin{array}{ccc}
\mathbb{1} & \mathbb{D} & \mathbb{0} \\
A & B_{w} & B
\end{array}\right]<} & \tau_{\mathrm{lft}}\left[\begin{array}{ccc}
C_{z} & D_{z w} & D_{z u} \\
\mathbb{O} & \mathbb{1} & \mathbb{0}
\end{array}\right]^{\prime}\left[\begin{array}{cc}
X_{\mathrm{lft}} & Y_{\mathrm{lft}} \\
Y_{\mathrm{ft}}^{\prime} & Z_{\mathrm{lft}}
\end{array}\right]\left[\begin{array}{ccc}
C_{z} & D_{z w} & D_{z u} \\
\mathbb{O} & \mathbb{1} & \mathbb{0}
\end{array}\right] \\
& +\tau\left[\begin{array}{lll}
K C & K D_{y w} & K D-\mathbb{1}
\end{array}\right]^{\prime}\left[\begin{array}{ccc}
K C & K D_{y w} & K D-\mathbb{1}
\end{array}\right]
\end{aligned}
$$

These inequalities imply for all non-zero vectors $\left(\begin{array}{ccc}x^{\prime} & w^{\prime} & u^{\prime}\end{array}\right)^{\prime} \neq 0$ :

$$
\begin{aligned}
& \left(\begin{array}{c}
x \\
A x+B_{w} w+B u
\end{array}\right)^{\prime}\left[\begin{array}{cc}
\mathbb{D} & P_{q} \\
P_{q} & \mathbb{0}
\end{array}\right]\left(\begin{array}{c}
x \\
A x+B_{w} w+B u
\end{array}\right) \\
& <\tau_{\mathrm{lft}}\left(\begin{array}{c}
C_{z} x+D_{z w} w+D_{z u} u \\
w
\end{array}\right)^{\prime}\left[\begin{array}{cc}
X_{\mathrm{lft}} & Y_{\mathrm{lft}} \\
Y_{\mathrm{lft}}^{\prime} & Z_{\mathrm{lft}}
\end{array}\right]\left(\begin{array}{c}
C_{z} x+D_{z w} w+D_{z u} u \\
w
\end{array}\right) \\
& +\tau\left(K C x+K D_{y w} w+K D u-u\right)^{\prime}\left(K C x+K D_{y w} w+K D u-u\right)
\end{aligned}
$$

Along the trajectories of $\left(\Sigma_{\mathrm{lft}} \stackrel{w, z}{\star} \Delta\right) \stackrel{u, y}{\star} K$ the vectors $x, w$ and $u$ are such that:

$$
\dot{x}=A x+B_{w} w+B u \quad, \quad u=K y=K C x+K D_{y w} w+K D u \quad, \quad z=C_{z} x+D_{z w} w+D_{z u} u \quad, \quad w=\Delta z
$$

Therefore, along the trajectories of the system, one gets that:

$$
\dot{x}^{\prime} P_{q} x+x^{\prime} P_{q} \dot{x}<z^{\prime}\left[\begin{array}{ll}
\mathbb{1} & \Delta^{\prime}
\end{array}\right]\left[\begin{array}{cc}
X_{\mathrm{lft}} & Y_{\mathrm{lft}} \\
Y_{\mathrm{lft}}^{\prime} & Z_{\mathrm{lft}}
\end{array}\right]\left[\begin{array}{c}
\mathbb{1} \\
\Delta
\end{array}\right] z
$$

which is always negative since the uncertainty set is the $\left\{X_{\mathrm{lft}}, Y_{\mathrm{lft}}, Y_{\mathrm{lft}}\right\}$-ellipsoid. This proves the closed-loop stability for any matrix gain of the $\{X, Y, Z\}$-ellipsoid and for any $\left\{X_{\mathrm{lft}}, Y_{\mathrm{lft}}, Y_{\mathrm{lft}}\right\}$-dissipative uncertainty with the help of the unique Lyapunov function $V_{q}(x)=x^{\prime} P_{q} x$, i.e. both the quadratic stability and the quadratic resilience are simultaneously achieved. 


\section{B. Proof of necessity in theorem 4}

Assume $K$ is a robustly stabilising gain and define the closed-loop uncertain model:

$$
\Sigma_{\mathrm{lft}} \stackrel{u, y}{\star} K:\left\{\begin{array}{l}
\dot{x}(t)=A(K) x(t)+B_{w}(K) w(t) \\
z(t)=C_{z}(K) x(t)+D_{z w}(K) w(t)
\end{array}\right.
$$

In [PEA 98a], the robust stability with respect to the non-structured $\left\{X_{\mathrm{lft}}, Y_{\mathrm{lft}}, Y_{\mathrm{lft}}\right\}$-dissipative uncertainty is proved to be equivalent to the quadratic stability and writes as:

$\exists \boldsymbol{\tau}_{\mathbf{l f t}}>0, \quad \exists \mathbf{P}_{\mathbf{q}}>\mathbb{0}$
$\left[\begin{array}{cc}\mathbb{1} & \mathbb{0} \\ A(K) & B_{w}(K)\end{array}\right]^{\prime}\left[\begin{array}{cc}\mathbb{D} & \mathbf{P}_{\mathbf{q}} \\ \mathbf{P}_{\mathbf{q}} & \mathbb{0}\end{array}\right]\left[\begin{array}{cc}\mathbb{1} & \mathbb{0} \\ A(K) & B_{w}(K)\end{array}\right]<\boldsymbol{\tau}_{\mathbf{l f t}}\left[\begin{array}{cc}C_{z}(K) & D_{z w}(K) \\ \mathbb{O} & \mathbb{1}\end{array}\right]^{\prime}\left[\begin{array}{cc}X_{\mathrm{lft}} & Y_{\mathrm{lft}} \\ Y_{\mathrm{lft}}^{\prime} & Z_{\mathrm{lft}}\end{array}\right]\left[\begin{array}{cc}C_{z}(K) & D_{z w}(K) \\ \mathbb{0} & \mathbb{1}\end{array}\right]$

This amounts to:

$$
\begin{aligned}
\left(\begin{array}{c}
x \\
w \\
u
\end{array}\right)^{\prime}\left[\begin{array}{ccc}
\mathbb{1} & \mathbb{D} & \mathbb{0} \\
A & B_{w} & B
\end{array}\right]^{\prime}\left[\begin{array}{cc}
\mathbb{D} & \mathbf{P}_{\mathbf{q}} \\
\mathbf{P}_{\mathbf{q}} & \mathbb{0}
\end{array}\right]\left[\begin{array}{ccc}
\mathbb{1} & \mathbb{0} & \mathbb{0} \\
A & B_{w} & B
\end{array}\right]\left(\begin{array}{c}
x \\
w \\
u
\end{array}\right) \\
<\boldsymbol{\tau}_{\mathrm{lft}}\left(\begin{array}{c}
x \\
w \\
u
\end{array}\right)^{\prime}\left[\begin{array}{ccc}
C_{z} & D_{z w} & D_{z u} \\
\mathbb{0} & \mathbb{1} & \mathbb{0}
\end{array}\right]^{\prime}\left[\begin{array}{cc}
X_{\mathrm{lft}} & Y_{\mathrm{lft}} \\
Y_{\mathrm{lft}}^{\prime} & Z_{\mathrm{lft}}
\end{array}\right]\left[\begin{array}{ccc}
C_{z} & D_{z w} & D_{z u} \\
\mathbb{0} & \mathbb{1} & \mathbb{0}
\end{array}\right]\left(\begin{array}{c}
x \\
w \\
u
\end{array}\right)
\end{aligned}
$$

for all non zero vectors $\left(\begin{array}{lll}x^{\prime} & w^{\prime} & u^{\prime}\end{array}\right) \neq 0$ such that:

$$
\left[\begin{array}{ccc}
K C & K D_{y w} & K D-\mathbb{1}
\end{array}\right]\left(\begin{array}{l}
x \\
w \\
u
\end{array}\right)=0
$$

Applying Finsler's lemma, it implies that a scalar $\tau$ exists such that:

$$
\begin{aligned}
{\left[\begin{array}{ccc}
\mathbb{1} & \mathbb{1} & \mathbb{0} \\
A & B_{w} & B
\end{array}\right]^{\prime}\left[\begin{array}{cc}
\mathbb{0} & \mathbf{P}_{\mathbf{q}} \\
\mathbf{P}_{\mathbf{q}} & \mathbb{D}
\end{array}\right]\left[\begin{array}{ccc}
\mathbb{1} & \mathbb{0} & \mathbb{0} \\
A & B_{w} & B
\end{array}\right]<} & \boldsymbol{\tau}_{\mathbf{l f t}}\left[\begin{array}{ccc}
C_{z} & D_{z w} & D_{z u} \\
\mathbb{0} & \mathbb{1} & \mathbb{D}
\end{array}\right]^{\prime}\left[\begin{array}{cc}
X_{\mathrm{lft}} & Y_{\mathrm{lft}} \\
Y_{\mathrm{lft}}^{\prime} & Z_{\mathrm{lft}}
\end{array}\right]\left[\begin{array}{ccc}
C_{z} & D_{z w} & D_{z u} \\
\mathbb{O} & \mathbb{1} & \mathbb{D}
\end{array}\right] \\
& +\boldsymbol{\tau}\left[\begin{array}{lll}
K C & K D_{y w} & K D-\mathbb{1}
\end{array}\right]^{\prime}\left[\begin{array}{ccc}
K C & K D_{y w} & K D-\mathbb{1}
\end{array}\right]
\end{aligned}
$$

which corresponds to the inequality in (15) with:

$$
\mathbf{X}=\tau K^{\prime} K \quad \mathbf{Y}=-\tau K^{\prime} \quad \mathbf{Z}=\tau \mathbb{1}
$$

Take the bottom right block of the last inequality, it writes as:

$$
\mathbb{0}<\tau_{\mathrm{lft}} D_{z u}^{\prime} X_{\mathrm{lft}} D_{z u}+\tau(D K-\mathbb{1})^{\prime}(D K-\mathbb{1})
$$

The matrix $X_{\mathrm{lft}}$ is assumed to be negative semi-definite and therefore it implies, on the one hand, that the closed-loop is well defined ( $D K-\mathbb{1}$ is non singular) and on the other hand, that the scalar $\tau$ is strictly positive. Hence, the obtained matrix $\mathbf{Z}$ is definite positive and $\mathbf{X}=\mathbf{Y} \mathbf{Z}^{-1} \mathbf{Y}^{\prime}$. The non-linear constraint (6) holds.

\section{Proof of theorem 6}

The major part of the proof is omitted. It mainly follows the proof of theorem 4. Take $K$ and $\tau>0$ such that (24). Then, apply the non zero vector $\left(x^{\prime}(t) w^{\prime}(t) v_{\infty}^{\prime}(t) u^{\prime}(t)\right)^{\prime} \neq 0$ to inequality (18). It implies that along the trajectories of the closed-loop system:

$$
x^{\prime}(t) P_{\infty} \dot{x}(t)+\dot{x}^{\prime}(t) P_{\infty} x(t)<\tau_{\mathrm{lft} \infty} z^{\prime}(t)\left[\begin{array}{c}
\mathbb{1} \\
\Delta
\end{array}\right]^{\prime}\left[\begin{array}{cc}
X_{\mathrm{lft}} & Y_{\mathrm{lft}} \\
Y_{\mathrm{lft}}^{\prime} & Z_{\mathrm{lft}}
\end{array}\right]\left[\begin{array}{c}
\mathbb{1} \\
\Delta
\end{array}\right] z(t)-\tau_{\infty} g_{\infty}^{\prime}(t) g_{\infty}(t)+\tau_{\infty} \gamma_{\infty}^{2} v_{\infty}^{\prime}(t) v_{\infty}(t)
$$


The $\Delta$-dependent term is negative since the uncertainty set is the $\left\{X_{\mathrm{lft}}, Y_{\mathrm{lft}}, Z_{\mathrm{lft}}\right\}$-ellipsoid. If there is no perturbation input $\left(v_{\infty}(t)=0\right)$ the stability of the system is proved with a quadratic Lyapunov function $V(x)=$ $x^{\prime} P_{\infty} x$. Moreover, taking the time average, the inequality gives:

$$
\lim _{T \rightarrow \infty} \frac{1}{2 T} \int_{-T}^{T} \dot{V}(x(t)) d t+\tau_{\infty}\left\|g_{\infty}\right\|^{2}<\tau_{\infty} \gamma_{\infty}^{2}\left\|v_{\infty}\right\|^{2}
$$

With the usual assumptions on the vectors, [DOY 94], [ZHO 94], it yields $\left\|g_{\infty}\right\|<\gamma_{\infty}\left\|v_{\infty}\right\|$. The $H_{\infty}$ norm of the closed-loop system is bounded by $\gamma_{\infty}$.

\section{Proof of theorem 7}

It follows the same lines as proof of theorem 6. Take $K$ and $\tau>0$ such that (24). Then, apply the non zero vector $\left(x^{\prime}(t) w^{\prime}(t) u^{\prime}(t)\right)^{\prime} \neq 0$ to inequality (20). It implies that along the trajectories of the closed-loop system (the $\Delta$-dependent term is removed for the same reasons as in the proof of theorem 6 ):

$$
x^{\prime}(t) P_{2}\left(\dot{x}(t)-B_{2} v_{2}\right)+\left(\dot{x}-B_{2} v_{2}\right)^{\prime}(t) P_{2} x(t)+\tau_{2} g_{2}^{\prime}(t) g_{2}(t)<0
$$

If there is no perturbation input $\left(v_{2}(t)=0\right)$ the stability of the system is proved with a quadratic Lyapunov function $V(x)=x^{\prime} P_{2} x$. Moreover, take the time average as in [DOY 94], and assume $v_{2}$ is a white noise with unit spectral density, it yields:

$$
\tau_{2}\left\|g_{2}\right\|^{2}<\operatorname{Trace}\left(B_{2}^{\prime} \mathbf{P}_{2} B_{2}\right) \leq \tau_{2} \gamma_{2}^{2}
$$

The power norm of the output signal $g_{2}(t)$ is less than $\gamma_{2}$ when the disturbance input is a unitary white noise. The $H_{2}$ norm of the closed-loop system is bounded by $\gamma_{2}$. 


\section{CONTENTS}

I Introduction $\quad 1$

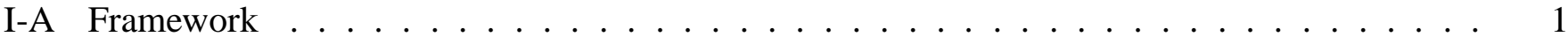

I-B Synthesis by quadratic separation . . . . . . . . . . . . . . . . . . . . 2

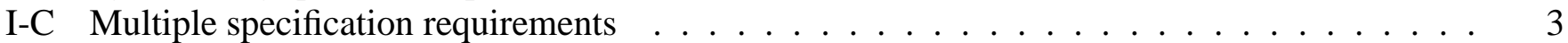

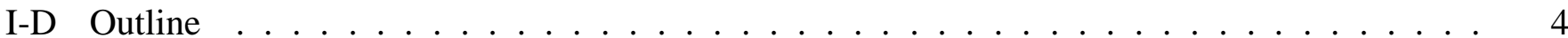

II Preliminaries $\quad 5$

II-A Notations . . . . . . . . . . . . . . . . . . . . . 5

II-B Matrix ellipsoid . . . . . . . . . . . . . . . . . . . . . 5

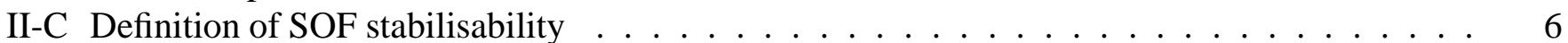

II-D Ellipsoid design for stabilisability . . . . . . . . . . . . . . . . . . 7

$\begin{array}{lr}\text { III Resilient stabilisability } & 8\end{array}$

III-A Definitions . . . . . . . . . . . . . . . . . . . . . . . . . . 8

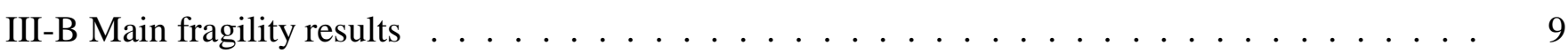

III-C Some types of control law uncertainties . . . . . . . . . . . . . . . . . . . 9

$\begin{array}{ll}\text { IV Robust stabilisability } & 10\end{array}$

IV-A Definitions . . . . . . . . . . . . . . . . . . . . . . . . 10

IV-B Robust stabilisability results . . . . . . . . . . . . . . . . . . . . 11

$\begin{array}{llr}V & \text { Controller design for robust performances } & 13\end{array}$

V-A Robust pole location . . . . . . . . . . . . . . . . . . . . . 13

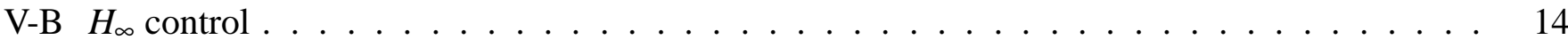

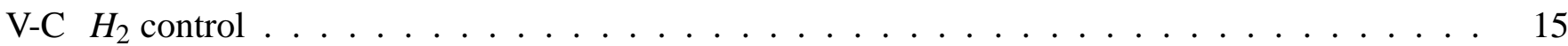

$\begin{array}{lr}\text { VI Complexity and conservatism } & 17\end{array}$

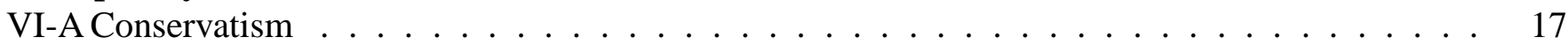

VI-B Multi-objective synthesis ． . . . . . . . . . . . . . . . . . . . . . . . . . 19

VI-C Numerical methods . . . . . . . . . . . . . . . . . . . . . . . . . . 20

$\begin{array}{lr}\text { VII Conclusions } & 21\end{array}$

$\begin{array}{ll}\text { References } & 21\end{array}$

VIIIProofs $\quad 24$

VIII-AProof of sufficiency in theorem $4 \ldots \ldots \ldots \ldots \ldots$

VIII-BProof of necessity in theorem $4 \ldots \ldots \ldots \ldots \ldots \ldots$

VIII-CProof of theorem $6 \ldots \ldots \ldots \ldots \ldots \ldots \ldots \ldots$

VIII-DProof of theorem $7 \ldots \ldots \ldots \ldots \ldots \ldots \ldots$ 